\title{
SINGULAR GEOMETRY OF THE MOMENTUM SPACE: FROM WIRE NETWORKS TO QUIVERS AND MONOPOLES
}

\author{
RALPH M. KAUFMANN, SERGEI KHLEBNIKOV, AND BIRGIT WEHEFRITZ-KAUFMANN
}

\begin{abstract}
A new nano-material in the form of a double gyroid has motivated us to study (non)-commutative $C^{*}$ geometry of periodic wire networks and the associated graph Hamiltonians.

Here we present a general more abstract framework, which is given by certain quiver representations, with special attention to the original case of the gyroid as well as related cases, such as graphene. The resulting effective $C^{*}$-geometry is that of the momentum space, which parameterizes the quasi-momenta.

This geometry is usually singular, where the singularities describe so-called band intersections in physics. We give geometric and algebraic methods to study these intersections; their origin being singularity theory and representation theory. A technique we newly apply to this situation is the use of topological invariants, which we formalize and explain in the paper. This uses $K$-theory and Chern classes as well as "slicing methods" for their computation. In this method the invariants can be computed using Berry's connection in the momentum space. This brings monopole charges and issues of topological stability into the picture.

Adding a constant magnetic field or allowing projective representations makes the $C^{*}$ geometry non-commutative. In this case, we can also use K-theory, albeit in a different way, to make statements about the band structure using gap labeling.
\end{abstract}

\section{INTRODUCTION}

Recently, a new nano-material in the form of a double gyroid has been synthesized [38]. It is based on a thickened triply-periodic minimal surface, whose complement consists of two non-intersecting channels. These can be filled with conducting or semiconducting materials [38] to function as nanowire networks with potentially useful electronic properties [26]. The nontrivial topology of such a network has motivated our study of its commutative and noncommutative geometry [21]. Following Bellissard and Connes [4, 9, 30], we proceed by identifying the relevant $C^{*}$-algebra, which in our case is spanned by the symmetries and the tight-binding (Harper) Hamiltonian of the skeletal graph obtained as a deformation retract of the channel. This approach leads to an effective geometry described by a family of finite dimensional Hamiltonians and their spectra; the latter determine the band structure of the original nanostructured solid in the tight-binding approximation.

In this paper, we analyze this effective geometry, which in condensed matter physics is called the momentum space and a cover of it, using methods from singularity theory and topology. The singularities of the geometry are of particular interest as they determine physical properties of the material. The most prominent example of this are the so-called Dirac points of graphene which lead to its amazing properties including room temperature quantum Hall effect [8]. In our setup, we show that these Dirac points can be thought of as pull backs of $A_{1}$ singularities for the particular case of the honeycomb lattice.

Singularities can also be forced by symmetries. One way to obtain such symmetries is via symmetries of the underlying graph/quiver. This construction is not direct and proceeds through several steps. The starting point is a re-gauging groupoid for matrix representations of the 
Hamiltonian. These are then "transferred" to actions on the base spaces of the effective geometry. The outcome is given by projective representations of subgroups of the symmetries of the graph on bundles over the subsets of the momentum space stabilized by the respective subgroups.

In this paper, we generalize the condensed matter setup to quiver representations stemming from finite graphs, thus making the theory more applicable to other contexts. In the process we adapt the techniques of [23] and [24] to this more general situation. In particular, we get a classification of singularities in the spectrum - the band intersections. The simplest of these is a conical intersection of two bands - these are the Dirac points mentioned above. We give analytic tools to compute locations and properties of all the singular points.

In the general framework above, we also give a formulation and application of the Berry phase phenomenon [7] in terms of $K$-theory and Chern-classes generalizing the observations of Thouless et al. (TKNN) [37] and Simon [34]. These concepts include topological charges in various guises: scalar, $K$-theoretic and cohomological. When the parameter space is threedimensional, isolated conical degeneracies are magnetic monopoles in the parameter space [7]. In the present case, the parameters are components of the crystal momentum $\mathbf{k}$; their number equals the dimensionality of the original periodic structure. Thus, in three spatial dimensions - the case of the gyroid - Dirac points are monopoles in the momentum space and, as we will see, are stable with respect to small deformations of the graph Hamiltonian. Furthermore, using foliations, we consider a slicing technique which leads to an effective numerical tool for finding singular points in the spectrum, generalizing the method used for this purpose in [40]. This technique has been implemented in [25] and corroborates the topological stability of the gyroid's Dirac points. This stability is not a common characteristic of all Dirac points: those of graphene, which is described by the honeycomb lattice, do not exhibit this property for general deformations.. There of course might be deformations which do preserve them see e.g. [12].

This fact has an elegant and short explanation in our approach. We expect that this analysis will contribute to the understanding of potential applications of gyroid-based nanomaterials, as well as to the theory of three-dimensional generalizations of the quantum Hall effect, along the lines of [5]. In two dimensions, the TKNN equations for generalized Dirac-Harper operators have been worked out in [29]. Analyses of higher-dimensional situations are contained in [11, 14, $6,27,15]$.

Even without going to complete generality provided by quiver representations, our approach to studying wire networks is not restricted to the gyroid system and applies to any embedded periodic wire network in $\mathbb{R}^{n}$. We have already used it to study more examples, namely, Bravais lattices, the honeycomb lattice and two other triply periodic surfaces and their wire networks, the primitive cubic (P surface) and the diamond (D surface). We refer to these as the geometric examples. We recall some results here and include a new consideration of the topological charges.

The effective $C^{*}$ geometry becomes non-commutative if we add an external magnetic field or more generally allow projective representations for the quivers. In the embedded wire network cases the noncommutative geometry is given by a subalgebra of a matrix algebra with coefficients in the noncommutative torus. Here the parameters of the torus correspond to the coefficients of the constant $B$-field that the material is subjected to.

One surprising fact is that some properties of the non-commutative situation are similar to the situation without a magnetic field, and there is evidence for duality between these two situations. The duality concerns the degenerate subspaces of the torus that appears as the relevant moduli space in both cases. In the commutative case, i.e. in the absence of a magnetic field, the torus is the base for the family of Hamiltonians and the requisite subspace is where the spectrum of the Hamiltonian has degeneracies. In the noncommutative case, the same torus parameterizes 
the $B$-field and the locus of degeneracy is that of those values of $B$ where the $C^{*}$-algebra is not the full matrix algebra.

The paper is organized as follows: In Chapter 1, we start with a description of the material that motivated this study and its underlying geometry. This is independent of the rest of the paper and may be skipped by the reader interested in the more general setup. The first chapter also discusses how the gyroid surface geometry is reduced to that of the skeletal graph- the deformation retract of a channel component of the complement to the triply periodic surface. We additionally introduce other related geometries which we consider in parallel. These are the honeycomb lattice underlying graphene, and the $\mathrm{P}$ and $\mathrm{D}$ surfaces, which are the other triply periodic self-symmetric surfaces.

Chapter 2 describes the mathematical model we work with. This includes the Harper Hamiltonian and the relevant Hilbert space and $C^{*}$ algebra for the commutative and non-commutative cases. We first consider the case of a periodic lattice embedded in $\mathbb{R}^{n}$ and then give the generalization to groupoid quiver representations in section 2.2. The reader more interested in the general mathematical framework can use this section as a starting point for reading the paper.

We discuss the resulting $C^{*}$ geometry in Chapter 3 . This includes the general setup identifying the singular locus as a pull-back from a miniversal unfolding, the representation theory using the re-gauging groupoid and our analysis of the Berry connection, topological charges and stability of the singular points as well as a slicing method to detect singular points or monopoles. Chapter 4 contains all results for the specific examples of the triply periodic wire systems $\mathrm{P}, \mathrm{D}$ and $\mathrm{G}$, as well as the two-dimensional honeycomb system and Bravais lattices in any dimension. This includes the new results about the topological charges. Using the methods of Chapter 3, we give the singular locus that is the degeneracies in the spectrum of the Harper Hamiltonian. As a second set of results, we review the classification results for the non-commutative geometries for the cases above. Here the parameter space is given by the background magnetic field 2-forms. In Chapter 5 we give a brief outlook including an observation of an almost duality.

\section{The Double Gyroid (DG) and Related Geometries and Material}

1.1. The Geometry. The gyroid is a triply periodic constant mean curvature surface that is embedded in $\mathbb{R}^{3}$ [16]. Figure 1 shows a picture of the gyroid. It was discovered in 1970 by Alan Schoen [33]. A single gyroid has symmetry group $I 4_{1} 32$ in Hermann-Maguin notation. Here the letter $I$ stands for bcc. The gyroid surface can be visualized by using the level surface approximation [28]

$$
L_{t}: \sin x \cos y+\sin y \cos z+\sin z \cos x=t
$$

In nature the single gyroid was observed as an interface for di-block co-polymers [18]. The double gyroid consists of two mutually non-intersecting embedded gyroids. Its symmetry group is $I a \overline{3} d$ where the extra symmetry comes from interchanging the two gyroids. It also has a level surface approximation which is given by the above expression (1) with $L_{w}$ and $L_{-w}$ for $0 \leq w<\sqrt{2}$. The picture on the left-hand side of Figure 1 is actually a double gyroid or a "thick" surface.

Let us fix some notation. We will denote by $S=S_{1} \amalg S_{2}$ the double gyroid surface. Its complement $C=\mathbb{R}^{3} \backslash S$ has three connected components, which we will call $C_{+}, C_{-}$and $W . W$ can be thought of as a "thickened" (fat) surface which we will refer to as DG wall. There is a deformation retract of $W$ onto a single gyroid.

There are also two channel systems $C_{+}$and $C_{-}$, shown in Figure 1. These channels form Yjunctions where three channels meet under a 120 degree angle. Each of these channel systems can 

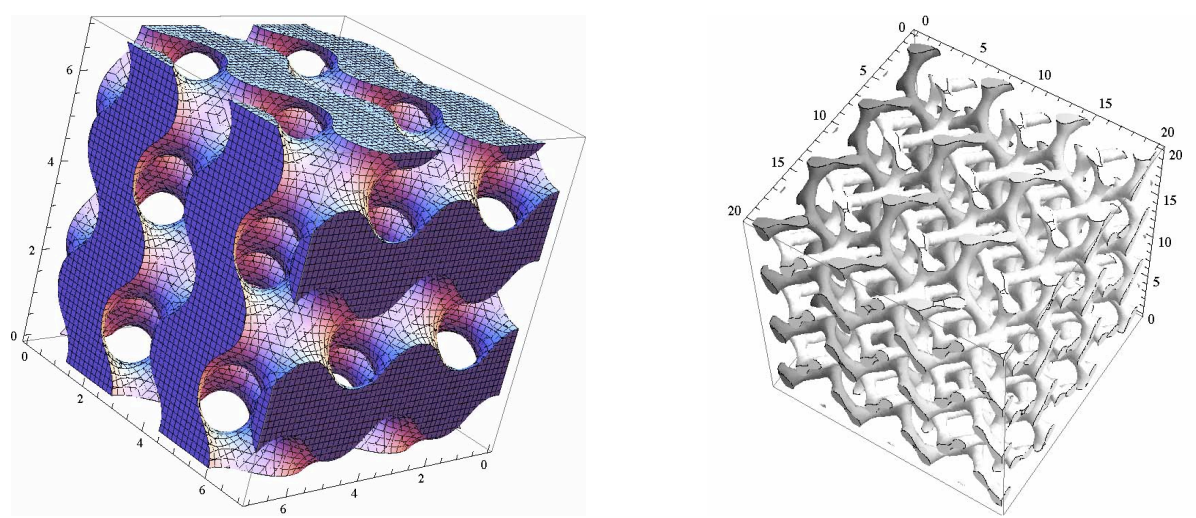

Figure 1 . The fat gyroid surface $W$ (left) and the two channel systems $C_{+}$ and $C_{-}$(right)

be deformation retracted to a skeletal graph $\Gamma_{ \pm}$. We will concentrate on one of these channels and its skeletal graph $\Gamma_{+}$, shown in Figure 2.
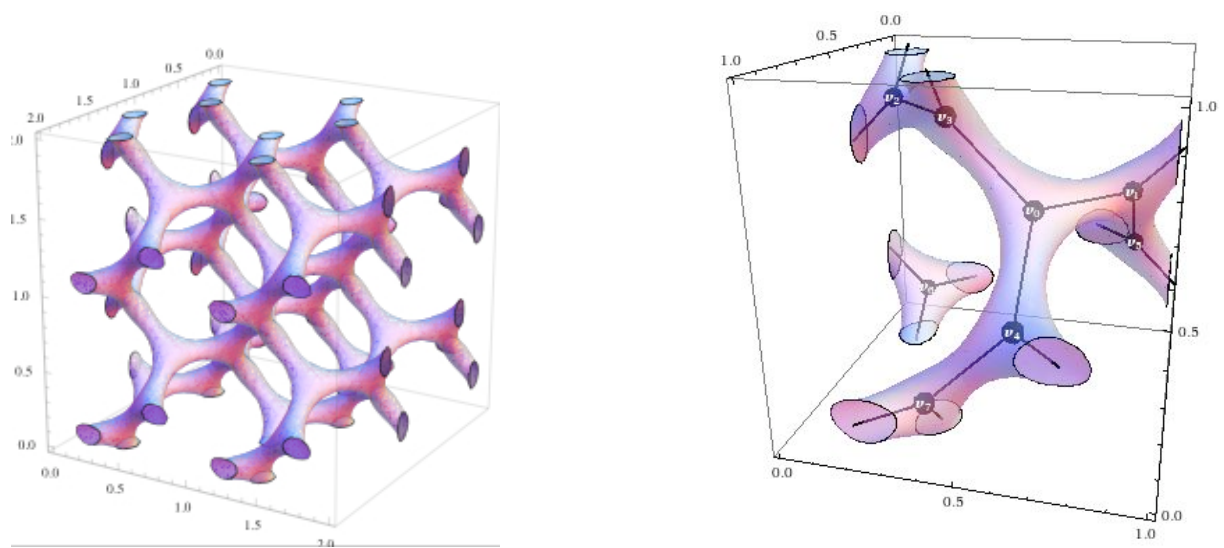

Figure 2. One of the two channels (left) and its skeletal graph in the unit cell (right)

1.2. The Material and Production. A solid-state double gyroid can be synthesized by selfassembly at the nanoscale, as demonstrated by Urade et al. [38]. The first step is production of a nanoporous silica film with the structure of unidirectionally cotracted double gyroid (DG) with lattice constant of about $18 \mathrm{~nm}$. The pores in the structure can then be filled with other materials to form nanowires. Fabrication of platinum DG nanowires by electrodeposition has been demonstrated in [38], where it has also been mentioned that the process can be used for other metals or semiconductors.

1.3. Related Geometries: the $\mathbf{P}$ and $\mathbf{D}$ surfaces. There are two other triply periodic selfdual and symmetric CMC surfaces- the cubic (P) and the diamond (D) network. They are shown in Figure 3 together with their wire networks obtained in the same way as for the gyroid. Here we summarize the results from [22]. 
The $\mathrm{P}$ surface has a complement which has two connected components each of which can be retracted to the simple cubical graph whose vertices are the integer lattice $\mathbb{Z}^{3} \subset \mathbb{R}^{3}$. The translational group is $\mathbb{Z}^{3}$ in this embedding, so it reduces to the case of a Bravais lattice.

The D surface has a complement consisting of two channels each of which can be retracted to the diamond lattice $\Gamma_{\diamond}$. The diamond lattice is given by two copies of the fcc lattice, where the second fcc is the shift by $\frac{1}{4}(1,1,1)$ of the standard fcc lattice, see Figure 3 . The edges are nearest neighbor edges. The symmetry group is $F d \overline{3} m$.
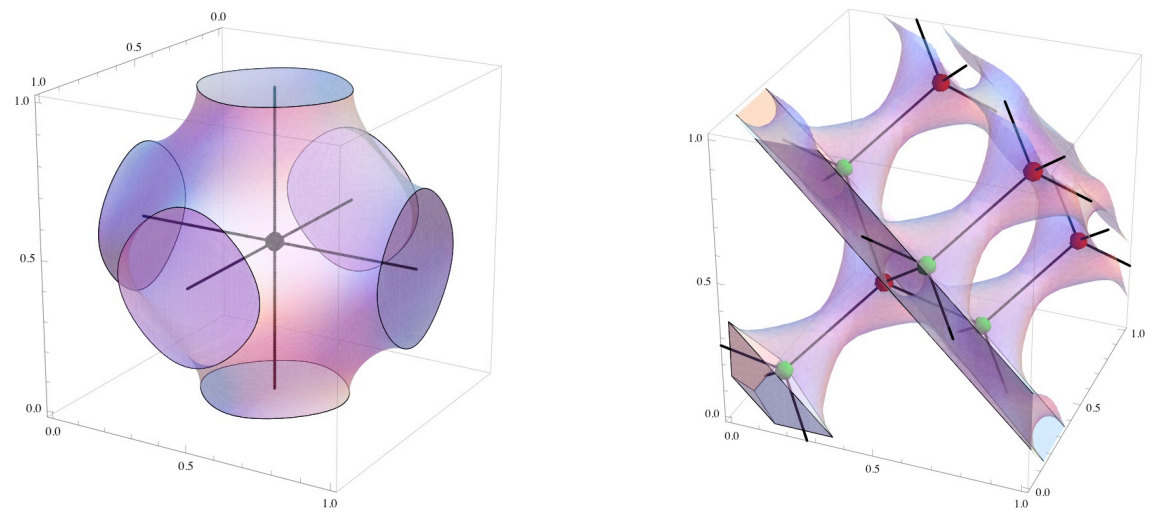

Figure 3. The cubic (P) (left) and the diamond (D) wire network (right)

1.4. Graphene. Graphene consists of one-atom thick planar sheets of carbon atoms that are densely packed in a honeycomb crystal lattice. This two-dimensional material has attracted much interest recently, partially because of the existence of Dirac points where excitations show a linear dispersion relation. Its electronic properties are described by a Harper Hamiltonian: see the review [8] and references therein. Here we will reproduce some of the known facts, such as the Dirac points using our non-commutative geometry machine.

\section{Mathematical Model and Generalization: Graphs and Groupoid REPRESENTATION}

2.1. Discrete model and Harper Hamiltonian. We will now describe how to obtain the Harper Hamiltonian for any given graph $\Gamma \in \mathbb{R}^{n}$ with a given maximal translation group $L \simeq \mathbb{Z}^{n}$ [19]. We will start with the commutative case without an external field, and then progress to the non-commutative case where the graph is placed in a constant external magnetic field. The mathematical set-up we will describe below can be understood in terms of Weyl quantization and Peierls substitution in physics [32]. Without the magnetic field the Harper Hamiltonian is given by translations, but in the presence of a magnetic field all translations turn into magnetic translations or Wannier operators, which cease to commute with each other.

Mathematically the discretization by the above process yields the Hilbert space $\mathscr{H}=\ell^{2}(V(\Gamma))$, where $V(\Gamma)$ are the vertices of $\Gamma$, and a projective representation of the translation group $L$ as well as an operator $H$, the Harper Hamiltonian. Concretely, the elements $l$ of $L$ act on the functions $\Psi$ via the usual translations $T_{l}: T_{l} \Psi\left(l^{\prime}\right)=\Psi\left(l-l^{\prime}\right)$.

2.1.1. Quotient Graph and Harper Hamiltonian. In general, given an embedded graph $\Gamma \in \mathbb{R}^{n}$, with a given maximal translation group $L \simeq \mathbb{Z}^{n}$, we consider the quotient graph $\bar{\Gamma}:=\Gamma / L$ and the projection $\pi: \Gamma \rightarrow \bar{\Gamma}$. The quotient graphs for our four main examples are given in Figure 4. 


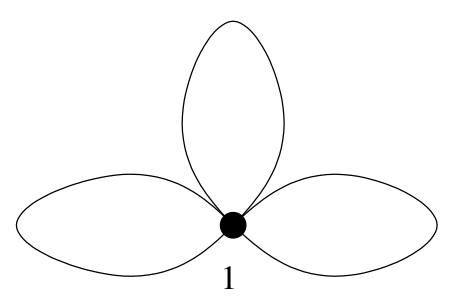

P

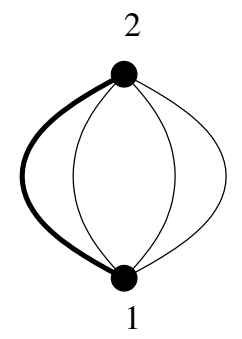

D

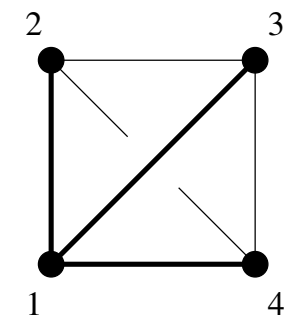

G

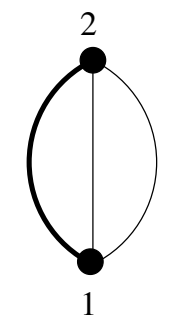

honeycomb

FiguRE 4. The quotient graphs of the P,D,G surfaces and the honeycomb lattice, together with a spanning tree and an order of the vertices.

The vertices of this graph are in 1-1 correspondence with vertices or sites of $\Gamma$ in a fundamental cell. We can think of the graph $\bar{\Gamma}$ as embedded into $T^{n}=\mathbb{R}^{n} / \mathbb{Z}^{n}$. Each edge $e$ of $\bar{\Gamma}$ lifts to a pair of edge vectors $\vec{e}, \overleftarrow{e}=-\vec{e}$ where the underlying line segment is any lift of $e$ to $\Gamma$. This is well defined since any two lifts differ by a translation.

To each vertex $v \in \bar{\Gamma}$ we can associate the Hilbert space $\mathscr{H}_{v}:=\ell^{2}\left(\pi^{-1}(v)\right)$. Then the whole Hilbert space $\mathscr{H}$ decomposes as

$$
\mathscr{H}=\bigoplus_{v \text { vertex of } \bar{\Gamma}} \mathscr{H}_{v}
$$

Since all the $\mathscr{H}_{v}$ are separable Hilbert spaces, they are all isomorphic.

The Harper Hamiltonian is then given as follows. For each edge $e$ between two vertices $v$ and $w$ of $\bar{\Gamma}$ let $T_{\vec{e}}$ be the translation operator from $\mathscr{H}_{w} \rightarrow \mathscr{H}_{v}$. This extends to an operator $\hat{T}_{\vec{e}}$ on $\mathscr{H}$ via $\hat{T}_{\vec{e}}=i_{\bar{v}} T_{\vec{e}} P_{\bar{w}}$ where $i_{\bar{v}}: \mathscr{H}_{\bar{v}} \rightarrow \mathscr{H}$ is the inclusion and $P_{\bar{w}}: \mathscr{H} \rightarrow \mathscr{H}_{\bar{w}}$ is the projection.

The Harper Hamiltonian is

$$
H=\sum_{e \text { edges of } \bar{\Gamma}} \hat{T}_{\vec{e}}+\hat{T}_{-\vec{e}}
$$

2.1.2. Harper Hamiltonian in the presence of a magnetic field. Adding a constant magnetic field requires a slightly different definition of the Harper Hamiltonian. We will use projective translation operators whose commutators include the fluxes of the magnetic field as follows: We define a 2-cocycle $\alpha_{B} \in Z^{2}(L, U(1))$ by a two-form $\hat{\Theta}$ on the ambient $\mathbb{R}^{n}$. Such a two-form is given by a skew symmetric matrix $\Theta$ with $\hat{\Theta}=\Theta_{i j} d x_{i} \wedge d x_{j}$. We let $B=2 \pi \hat{\Theta}$ and interpret it as a quadratic form ${ }^{1}$. In this way we obtain a two-cocycle

$$
\alpha_{B} \in Z^{2}\left(\mathbb{R}^{n}, U(1)\right): \alpha_{B}(u, v)=\exp \left(\frac{i}{2} B(u, v)\right)
$$

which we then restrict to $L$.

We define magnetic translations by starting from $A$, which is a potential for $B$ (on $\mathbb{R}^{n}$ ). The magnetic translation partial isometry is now acting on a wave function as

$$
U_{l^{\prime}} \psi(l)=e^{-i \int_{l}^{\left(l-l^{\prime}\right)} A} \psi\left(l-l^{\prime}\right)
$$

\footnotetext{
${ }^{1}$ This is the quadratic form on constant vector fields, which can be identified with a quadratic form on $\mathbb{R}^{n}$. The matrix $\Theta$ is also the matrix for this quadratic form.
} 
The magnetic Harper operator is defined as

$$
H=\sum_{e \text { edges of } \bar{\Gamma}} U_{\vec{e}}+U_{\overleftarrow{e}}
$$

2.2. Generalization: Groupoid and quiver representations. In the setting above, which we call the geometric examples, we have distilled the following data: a finite graph $\bar{\Gamma}$, the translational groups $L$ and a projective representation of it on $\mathscr{H}=\bigoplus \mathscr{H}_{v}$ and finally the Hamiltonian $H$.

We will now explore the possibility of obtaining such data from a more general setup. There are two ways to do this: in terms of groupoids or in terms of quivers.

2.2.1. Groupoid representation. Recall that a groupoid is a category whose morphisms are all invertible. A representation of a groupoid is a functor $\rho$ from this category into a linear category. In our case this will be the category of separable Hilbert spaces which is the full subcategory of the category of vector spaces whose objects are separable Hilbert spaces.

A graph $\bar{\Gamma}$ (here $\bar{\Gamma}$ need not be finite) determines a groupoid $\mathcal{G}$ as follows. The objects are the vertices of $\Gamma$. The morphisms are generated by the edges. That is for each oriented edge between $v$ and $w$ there is one generator $\phi_{\vec{e}}$ in $\operatorname{Hom}(v, w)$. The morphisms in this category are then the composable words in the $\phi_{\vec{e}}$ where composable means that the source of a latter is the target of the predecessor, with the relations that

$$
\phi_{\vec{e}} \phi_{\overleftarrow{e}}=i d_{v} \in \operatorname{Hom}(v, v) \text {, the identity element }
$$

What this means is that the morphisms are the paths on $\Gamma$ up to homotopy, with the constant path yielding the identity.

A groupoid representation of $\mathcal{G}$ in separable Hilbert spaces then assigns to each vertex $v$ of $\bar{\Gamma}$ a separable Hilbert space $\rho(v)=\mathscr{H}_{v}$ and to each oriented edge $\vec{e}$ from $v$ to $w$ a morphism $\rho\left(\phi_{\vec{e}}\right)=\Phi_{\vec{e}} \in \operatorname{Hom}\left(\mathscr{H}_{v}, \mathscr{H}_{w}\right)$ with the relation that $\Phi_{\vec{e}} \Phi_{\overleftarrow{e}}=i d_{\mathscr{H}_{v}}$. We will abbriviate $\rho\left(\phi_{\vec{e}}\right)$ by $\rho(\vec{e})$.

The groupoid representation is unitary if all the $\Phi_{\vec{e}}$ are.

Remark 2.1. Notice that there is an involution $*$ on the morphisms, by transposing the word and reversing the orientation of each letter. So we can only look at involutive functors, that is functors which send $*$ to $\dagger$, that is the Hermitian adjoint. This guarantees that the representation is unitary.

2.2.2. Quiver representation. There is a way to formulate this in quiver language. Given a graph $\bar{\Gamma}$ and an arbitrary choice of directions for the edges determines a quiver. Now one can construct the double of the quiver, where each oriented edge is doubled with reverse orientation. If we started from a graph, this means that each unoriented edge $e$ is replaced by the two oriented edges $\vec{e}$ and $\overleftarrow{e}$. Now the double of the quiver is independent of the original choice of orientation. As above, there is an involution $*$ on the set of its oriented edges which is given by reversing the orientation. We will restrict the quiver representations we consider to those where the involution $*$ goes to $\dagger$.

2.2.3. Hamiltonian of the representation. Just as above we set $\mathscr{H}:=\bigoplus_{v}$ vertex of $\bar{\Gamma} \mathscr{H}_{v}$ and define

$$
H:=\sum_{e \text { edges of } \bar{\Gamma}} \rho(\vec{e})+\rho(\overleftarrow{e}): \mathscr{H} \rightarrow \mathscr{H}
$$


2.2.4. Representation of $\pi_{1}(\bar{\Gamma})$. If we fix a vertex $v_{0}$ of $\bar{\Gamma}$, the groupoid representation naturally gives a representation of $\pi_{1}\left(\bar{\Gamma}, v_{0}\right)$ as follows. We fix a set of symmetric generators of $\pi_{1}\left(\bar{\Gamma}, v_{0}\right)$ which is isomorphic to the free group in $b_{1}=1-\chi$ generators $\mathbb{F}_{b_{1}}$, where $\chi=$ \#vertices - \#edges is the Euler characteristic and $b_{1}$ is the first Betti number. Each such generator $g_{i}$ is a directed simple loop on the graph which is given by a sequence of directed edges $\vec{e}_{1 i}, \ldots, \vec{e}_{n_{i} i}$. Then $\rho\left(g_{i}\right)=\rho\left(\vec{e}_{1 i}\right) \circ \cdots \circ \rho\left(\vec{e}_{n_{i} i}\right)$ gives a representation of $\pi_{1}\left(\bar{\Gamma}, v_{0}\right)$ on $\mathscr{H}_{v_{0}}$.

Definition 2.2. We will denote the algebra generated by $\rho\left(\pi_{1}\right)$ by $\mathscr{T}$. We say $\rho$ is maximal if the generators of $\pi_{1}$ map to linearly independent operators and we say that $\rho$ is of torus type if $\mathscr{T}=\mathbb{T}_{\Theta}^{n}$, the non-commutative $n$-torus with parameters given by the skew-symmetric matrix $\Theta$. Here necessarily $n=1-\chi(\Gamma)$.

If $\rho$ is of torus type then representation $\rho$ of $\pi_{1}\left(\bar{\Gamma}, v_{0}\right)=\mathbb{F}_{b_{1}}$ as a projective representation factors through $H_{1}(\bar{\Gamma})=\mathbb{Z}^{b_{1}}=\mathbb{F}_{b_{1}} /\left[\mathbb{F}_{b_{1}}, \mathbb{F}_{b_{1}}\right]$, the Abelianization of $\pi_{1}$. In the geometric setup of nano-wire networks, these are given by a constant background $B$ field and the parameter $\Theta$ is the matrix corresponding to that field as discussed above; see [21] for additional details.

In the geometric situation of Chapter 1, maximality is equivalent to the fact that the translational symmetry group is maximal.

2.2.5. Spanning trees. If we pick a rooted spanning tree of $\bar{\Gamma}$ then we get isomorphisms $\phi_{0 v}: \mathscr{H}_{v_{0}} \simeq \mathscr{H}_{v}$ by using $\rho$ and concatenation along the unique shortest path of oriented edges from $v_{0}$ to $v$ in the spanning tree. Let $\Phi=\bigoplus_{v} \phi_{0 v}: \mathscr{H}_{v_{0}}^{|V|} \rightarrow \mathscr{H}$. Then this isomorphism yields a representation $\tilde{\rho}$ on $\mathscr{H}_{v_{0}}^{|V|}$ via pullback.

Likewise $\phi_{v 0}$ induces an isomorphism of $\pi_{1}(\bar{\Gamma}, v)$ and $\pi_{1}\left(\bar{\Gamma}, v_{0}\right)$. Using this identification, we get a representation $\hat{\rho}$ of $\mathscr{T}$ on $\mathscr{H}$ and via pull-back with $\Phi$ on $\mathscr{H}_{v_{0}}^{|V|}$.

A rooted spanning tree $\left(\tau, v_{0}\right)$ also gives rise to one more bijection. This is between a set of (symmetric) generators of $\pi_{1}$ and the edges not in the spanning tree. The bijection is as follows.

If $\vec{e}$ is a directed edge from $v$ to $w$ then there is a generator $g_{\vec{e}}$ which is given by the following path of ordered edges: (1) the unique shortest path in $\tau$ from $v_{0}$ to $v(2)$ the directed edge $\vec{e}$ and (3) the unique shortest path in $\tau$ from $w$ to $v_{0}$. It is clear that $g_{\vec{e}}=g_{\overleftarrow{e}}^{-1}$. By contracting the spanning tree, we see that this is indeed a set of symmetric but otherwise independent generators.

For convenience, we set $g_{\vec{e}}=1$ if $e \in \tau$.

2.2.6. Quiver $C^{*}$-Geometry, the algebra $\mathscr{B}$. Given a groupoid representation in separable Hilbert spaces of a finite graph $\bar{\Gamma}$ we call the $C^{*}$ algebra generated by the operators $H$ and $\mathscr{T}$ via $\hat{\rho}$ on $\mathscr{H}$ the Bellissard-Harper algebra of the pair $(\bar{\Gamma}, \rho)$ and denote it by $\mathscr{B}$.

This general set gives the generalization of one of the results of [21].

Theorem 2.3. Any choice of spanning tree together with an order on the vertices gives rise to a faithful matrix representation of $\mathscr{B}$ in $M_{|V|}(\mathscr{T})$.

Proof. This follows from the fact that under $\Phi, \rho(\vec{e})$ gets transformed to the matrix entry $\rho\left(g_{\vec{e}}\right)$ between the copies of $\mathscr{H}_{v_{0}}$ corresponding to $\mathscr{H}_{v}$ and $\mathscr{H}_{w}$ under $\Phi$. Enumerating these vertices yields a matrix.

In the following given a rooted spanning tree $\tau$ we will only choose orders $<$ such that the root is the first element. The resulting matrix Hamiltonian will be denoted by $H_{\tau,<}$. 


\section{3. $C^{*}$-GEOMETRY}

3.1. Structure theorems for the $C^{*}$-geometry. The non-commutative $C^{*}$-geometry of such a quiver representation in general and the one stemming from the geometric situation in particular is that of $\mathscr{B}$.

Just like in $[21,22]$ one can now ask the question whether or not $\mathscr{B}$ is isomorphic to the full matrix algebra and hence Morita equivalent to $\mathscr{T}$ itself. In the geometric case, we obtain a family of algebras depending on a choice of magnetic field. To take this into account, we will denote by $\mathscr{B}_{\Theta}$ the resulting matrix algebra for the choice of magnetic field determined by $\Theta$. In this notation, $\mathscr{T}_{\Theta}=\mathbb{T}_{\Theta}$ is generically simple and led to the expectation - which we proved in [21] — that generically $\mathscr{B}_{\Theta}=M_{|V|}\left(\mathbb{T}_{\Theta}\right)$. This of course need not be the case in general.

It stands to reason that other more complicated physical phenomena could be described by such algebras.

It actually turns out that in the geometric examples not only is the algebra indeed the full matrix algebra at generic irrational parameter values, but that there are even only finitely many or at most a co-dimension-2 subset of matrix parameters $\Theta$, where $\mathscr{B}_{\Theta} \subsetneq M_{k}\left(\mathbb{T}_{\Theta}\right)$.

Theorem 3.1. [21, 22] For the geometric cases of the G surface and the honeycomb lattice the algebra $\mathscr{B}_{\Theta}$ is isomorphic to the full matrix algebra except at finitely many values of $\Theta$ given in Chapter 4. For the P surface and all Bravais lattices $\mathscr{B}_{\Theta}=\mathscr{T}_{\Theta}=M_{1}\left(\mathbb{T}_{\Theta}\right)$. For the $D$ surface, the set of values of $\Theta$ for which $\mathscr{B}_{\Theta} \subsetneq M_{2}\left(\mathbb{T}_{\Theta}\right)$ is given by 6 one dimensional families and finitely many special points (also listed in Chapter 4). If $\mathscr{B}_{\Theta}$ is the full matrix algebra then it is Morita equivalent to $\mathbb{T}_{\Theta}$.

Remark 3.2. Note that except for the $\mathrm{P}$ and general Bravais case, these families above give examples of continuos variations of algebras whose $K$-theory does not vary continuously. In those cases the $K$-theory for the commutative case $\Theta=0$, which corresponds to a nontrivial ramified cover of the torus $[21,22]$, is different from the generic situation, which has the $K$-theory of the torus. There are also certain special points where the algebra and hence the $K$-theory is isomorphic to the commutative case, although the magnetic field is not 0 . This happens if the magnetic flux through the relevant cells is integer. We also expect the $K$-theory to drop at the other special points, due to the presence of additional symmetries.

3.1.1. Inspecting the spectrum via K-theory labeling. One application of the non-commutative approach is gap labeling by $K$-theory. If the Hamiltonian $H$ has a spectrum bounded from below, then each gap in the spectrum gives rise to a projector $P_{<E}$ onto the Eigenspaces with Eigenvalues less than any fixed value $E$ in the gap, see e.g. [4, 30]. The gap labeling then associates the $K$-theory class of $P_{<E}$ to the gap.

By the above result, via the inclusion $\mathscr{B} \hookrightarrow M_{k}(\mathscr{T})$ the projector $P_{<E}$ also gives rise to a $K$-theory class in $K\left(M_{k}(\mathscr{T})\right) \simeq K(\mathscr{T})$. Using this embedding, one can deduce analogues of the famous Hofstadter Butterfly.

Theorem 3.3. If $(\bar{\Gamma}, \rho)$ is toric non-degenerate, then the Hamiltonian $H$ as an operator on $\mathscr{H}$ has only finitely many gaps if the magnetic field is rational in the sense that the matrix $\Theta$ is rational.

3.2. Effective geometry in the commutative case: the momentum space and the Eigenvalue cover. If $\mathscr{B}$ is commutative, for instance if $\Theta=0$ in the geometric situation, then by the Gel'fand-Naimark theorem, there is a compact ${ }^{2}$ Hausdorff space $X$, such that $\mathscr{B} \simeq C_{0}(X)$. The points of $X$ can be thought of as characters, i.e. $C^{*}$-homomorphisms $\chi: \mathscr{B} \rightarrow \mathbb{C}$. More

$2_{\mathscr{B}}$ is unital. 
precisely these characters are in bijection with the maximal ideals of $\mathscr{B}$ which are the points. If we wish to make this distinction, we write $p_{\chi}$ for the point of $X$ corresponding to the character $\chi$ and vice-versa $\chi_{p}$ for the character corresponding to $p$. Likewise there is a space $T$ which corresponds to the $C^{*}$-algebra $\mathscr{T}$. In the geometric case $T=T^{n}=\mathbb{R}^{n} / L$.

As usual the correspondence between the algebra of functions and the spaces is contravariant. This means that the inclusion $\hat{\rho}: \mathscr{T} \rightarrow \mathscr{B}$ gives rise to a morphism $\pi: X \rightarrow T$. If $(\rho, \Gamma)$ is maximal, then $\mathscr{T} \rightarrow \mathscr{B}$ is injective and hence $\pi: X \rightarrow T$ is surjective.

Furthermore let us consider the algebra $\mathscr{T}^{\oplus k}$ given by the direct sum of $k$ copies of $\mathscr{T}$. The space corresponding to this algebra is simply $T \amalg \cdots \amalg T k$-times.

Since after choosing an order and a rooted spanning tree $\mathscr{B} \subset M_{|V|}(\mathscr{T})$, we can lift any character $\chi$ of $\mathscr{T}$ to a $C^{*}$-homomorphism: $\hat{\chi}: M_{|V|}(\mathscr{T}) \rightarrow M_{|V|}(\mathbb{C})$ of $\mathscr{B}$ by applying $\chi$ to each entry.

Definition 3.4. We call a point $\chi$ of $T$ degenerate if $\hat{\chi}(H)$ has less than $|V|$ distinct Eigenvalues and we will denote this locus as $T_{d e g}$.

We also set $X_{\text {deg }}:=\pi^{-1}\left(T_{d e g}\right)$. These are the singular points of $X$.

Repeating the proof of [21] we arrive at the following

Theorem 3.5. If $(\rho, \bar{\Gamma})$ is maximal the map $\pi: X \rightarrow T$ is ramified over the degenerate points and furthermore $X$ is the quotient of the trivial $k$-fold cover of $T$ where the identifications are made in the fibers over degenerate points. Moreover these correspond to the degeneracies of $H$ over these points.

In other words, $X$ can be thought of as the spectrum of the family of Hamiltonians $H(p)=\chi_{p}(H)$ parameterized over $T$.

The key ingredient is the image of $H$ under the map $\mathscr{B} \rightarrow \mathscr{T}^{\oplus k}$ dual to the map $\amalg_{i=1}^{k} T \rightarrow X$

$$
H \mapsto \sum_{i} \lambda_{i} e_{i}
$$

where $e_{i}$ are the idempotents corresponding to the $\mathrm{i}$-th component and $\lambda_{i}$ is the $i$-th Eigenvalue.

\subsection{Singular geometry of the momentum space and the Eigenvalue cover.}

3.3.1. Characteristic map and Swallowtails. In the commutative case, the locus $X_{\mathrm{deg}}$ has a nice characterization in terms of singularity theory, [23]. First, there is an embedding of $X$ into $T \times \mathbb{R}$, where $X$ is identified with the pairs $\left(t, \lambda_{i}\right)$ for which $\lambda_{i}$ is an Eigenvalue of $H(t)$. Here $H(t)=\hat{\chi}_{t}(H)$, i.e. the point $t$ corresponds to the character $\chi_{t}$ under the Gel'fand representation.

The key ingredient is a newly defined characteristic map: for this let

$$
P(z, t)=\operatorname{det}(z I d-H(t))=z^{k}+b_{k-1}(t) z^{k-1}+\cdots+b_{0}(t),
$$

let

$$
P\left(z-\frac{b_{k-1}}{k}, z\right)=z^{k}+a_{k-2}(t) z^{k-2}+\cdots+a_{0}(t)
$$

and let $g$ be the isomorphism on $T \times \mathbb{R}$ which sends $(t, z)$ to $\left(t, z-\frac{b_{k-1}}{k}\right)$.

The coefficients $a_{k-2}(t), \ldots, a_{0}(t)$ define a map $\Xi: T \rightarrow \mathbb{C}^{k-1}$ called the characteristic map. We recall that the miniversal unfolding of the $A_{k-1}$ singularity $z^{k}$ is given by the family of functions $f_{a_{0}, \ldots, a_{k-2}}=z^{k}+a_{k-2} z^{k-2}+\ldots a_{1} z+a_{0}$, with the parameters $\left(a_{0}, \ldots, a_{k-2}\right) \in \mathbb{C}^{k-1}$ giving the base of this variation, see e.g. [2]. This is the base of the covers whose fiber over a point $\left(a_{0}, \ldots, a_{k-2}\right)$ are the roots of $f_{a_{0}, \ldots, a_{k-2}}$. The terms $z^{i}: 0 \leq i \leq k-2$ correspond to a basis of the Milnor or Jacobian ring $\mathbb{C}[z] /\left(z^{k-1}\right)$. It is miniversal in the sense that any other 
variation of resolving the singularity is a pull-back of a variation equivalent by diffeomorphism to this one. Notice that the term with $z^{k-1}$ is missing, which is why we used the map $g$ to reparameterize the characteristic equation.

Identifying $\mathbb{C}^{k-1}$ with the base of the miniversal unfolding of the $A_{k-1}$ singularity, we obtain the following (cf. [23]):

Theorem 3.6. The branched cover $X \rightarrow T$ is equivalent via $g$ to the pull back of the miniversal unfolding of the $A_{k-1}$ singularity along the characteristic map $\Xi$. Explicitly, if $\hat{P}=P \circ g$ then the pull back along $\Xi$ of the cover of the miniversal unfolding is the cover corresponding to the roots of $\hat{P}$. Via $g$ this cover is equivalent to the one of $P$ and hence to the cover $X$. Moreover if the family of Hamiltonians is traceless the cover is the pull-back on the nose.

The family is traceless if $\bar{\Gamma}$ has no small loops — that is edges which are a loop at one vertexand if, additionally, the graph is also simply laced, then $a_{k-2} \equiv|E(\bar{\Gamma})|$. In other words, the image of $T$ under $\Xi$ is contained in the corresponding slice $a_{k-2}=|E(\bar{\Gamma})|$ of the base of the miniversal unfolding.

This means that if $\Sigma \subset \mathbb{C}^{k-1}$ is the discriminant locus or swallowtail, then $T_{\text {deg }}=g^{-1}\left(\Xi^{-1}(\Sigma)\right)$ and the fiber of $\pi$ over a point $t$ is exactly $g^{-1} \pi_{A}^{-1}(\Xi(t))$ where $\pi_{A}$ is the projection of the miniversal unfolding.

Here the swallowtail $\Sigma$ is the set of points $\left(a_{0}, \ldots, a_{k-2}\right) \subset \mathbb{C}^{k-1}$ where $f_{a_{0}, \ldots, a_{k-2}}$ has roots of higher multiplicity - see Figure 5 for the $A_{2}$ and $A_{3}$ cases.

In other words the fibers over degenerate points are identified with the corresponding fibers over their image points in the swallowtail.

Using Grothendieck's characterization [10] of the swallowtail as stratified by lower order singularities obtained by deleting edges in the corresponding Dynkin diagram, and pulling this back via $\Xi$, we obtain:

Corollary 3.7. Consider a variation of Hamiltonians given by $(\bar{\Gamma}, \rho)$ and assume that the Hamiltonians are traceless. The only possible types of singularities in the spectrum of this variation are $\left(A_{r_{1}}, \ldots, A_{r_{s}}\right)$ with $1 \leq s \leq\lfloor k / 2\rfloor$, and $\sum_{i=1}^{s} r_{i} \leq k-s$.

In the simply laced case with no loops, $r_{i}<k$

Remark 3.8. Notice that our approach is "orthogonal" to the considerations of [13] where the projection $T \times \mathbb{C} \rightarrow \mathbb{C}$ was used instead of the projection $T \times \mathbb{C} \rightarrow T$ which we use. Also in their context, $T$ needs to be complex one-dimensional and hence their arguments do not generalize to arbitrary (odd and even) dimensions. This is because their theory relies on deep theorems which are special to the algebraic geometry of curves.

Remark 3.9. Theorem 3.6 and the corollary above can be viewed as a generalization/refinement of what is commonly referred to as the "von Neumann-Wigner theorem". This is not a theorem per se, but the expectation that for a "generic" variation the degenerate locus is of codimension 3. This goes back to the result of [39] that for the full family of all Hermitian Hamiltonians $\operatorname{Herm}(k)$ (the space of all $k \times k$ Hermitian matrices) the degenerate locus is indeed of codimension 3.

The most prominent results about the geometry of $\operatorname{Herm}(k)$ were already obtained in [39]. Here one can find the co-dimensions of the strata of degenerate Eigenvalues, basically by a dimension count. This was carried further in [3], where a fibration was introduced. For this and other discussions it is sometimes convenient to mod out the $k^{2}$-real-dimensional vector space $\operatorname{Herm}(k)$ by translations and dilatations. Indeed shifting the spectrum or scaling it does not change the topology of the situation. The translations are done by adding scalar matrices and the dilatation, as usual, by multiplying by non-zero constants. Modding out by the translations 

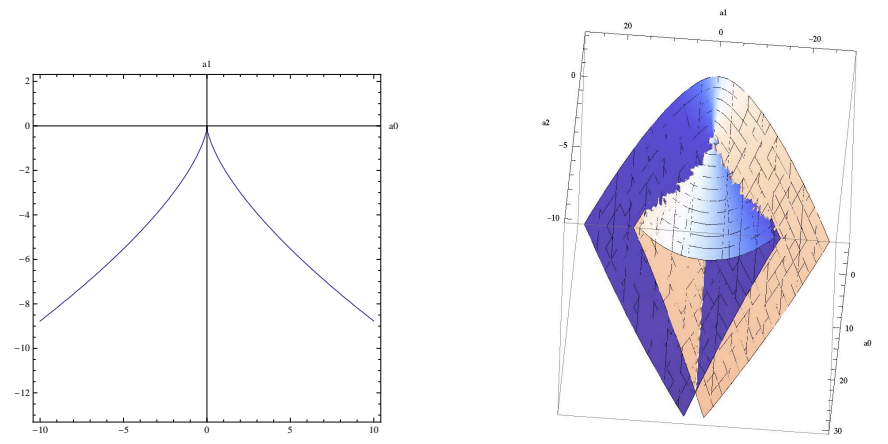

Figure 5 . The swallowtail for the $A_{2}$ (left) and $A_{3}$ (right) singularities

means that we can restrict to traceless matrices and modding out by dilatations means that we can take the norm to be 1 , unless we are dealing with the 0 matrix. The quotient space of the space of non-scalar Hermitian matrices under the simultaneous action, which is naturally identified with the co-invariants, is then a $k^{2}-2$-dimensional sphere which we denote by $\mathbb{S}(k)$. This sphere then has a filtration by pieces $F_{p}$ for which the first $p$ Eigenvalues are equal. Arnold [3] studied this filtration and that study has been continued in [1].

Our main focus is the geometry of a given (not necessarily generic) family $H: T \rightarrow H e r m(k)$ dictated by a quiver representation. In this setting the exact codimension depends on the whole family $T$ and is given precisely as the preimage of $\Xi$. To be more precise locally it is the dimension of the intersection of the image under $\Xi$ with the swallowtail and the dimension of the fiber.

Proposition 3.10. In the maximal toric case increasing the number of links to arbitrarily high values, the dimension of the degenerate locus $T_{\text {deg }}$ generically becomes $-\chi(\Gamma)$, so that the stable expected codimension of the degenerate locus is 1 .

Proof. Since the domain of $\Xi$ is compact, so is the image. Its size is limited by the coefficients of the Hamiltonian. The value of the $i, j$-th entry under a lifted character $\hat{\chi}$ is sharply bounded by $l$ where $l$ is the number of edges between $v_{i}$ and $v_{j}$. This is follows from to the definition of the Hamiltonian as translations along edges and is a generalization to many edges of [23] [Section 2 (equation (6)]. As the number of edges grows, this bound increases. This implies that the sharp bound on the coefficients $a_{i}$ also increases. If this is large enough, the image of $\Xi$ will fill out a bounded region of the complement of the swallowtail $\Sigma$ over which the discriminant is positive. Then the boundary of the image given by a part of the swallowtail $\Sigma$ will be of codimension 1 and of dimension $\left|V_{\Gamma}\right|-2$. The generic dimension of the fiber will be $\operatorname{dim}(T)-\left(\left|V_{\Gamma}\right|-1\right)$. In total this gives the dimension of the critical locus as $1-\chi(\Gamma)-\left|V_{\Gamma}\right|+1+\left|V_{\Gamma}\right|-2=-\chi(\Gamma)$.

The test case of the triangular graph with possibly multiple edges has been calculated in [22] which gives an example of the phenomenon described above. This is illustrated in Figure 6 and Figure 7 .

3.3.2. Characterizing Dirac points. Physically very interesting singularities of $X$ are conical singularities, which are also called Dirac points. In order to find these singularities, we consider the ambient space $T \times \mathbb{R}$ and the function $P: T \times \mathbb{R} \rightarrow \mathbb{R}$ as given in §3.3.1. As we argued in [23], Dirac points in the spectrum are isolated Morse singularities of $P$ with signature $(+,-, \ldots,-)$. That argument did not need the specifics of the geometric situation and hence generalizes. 

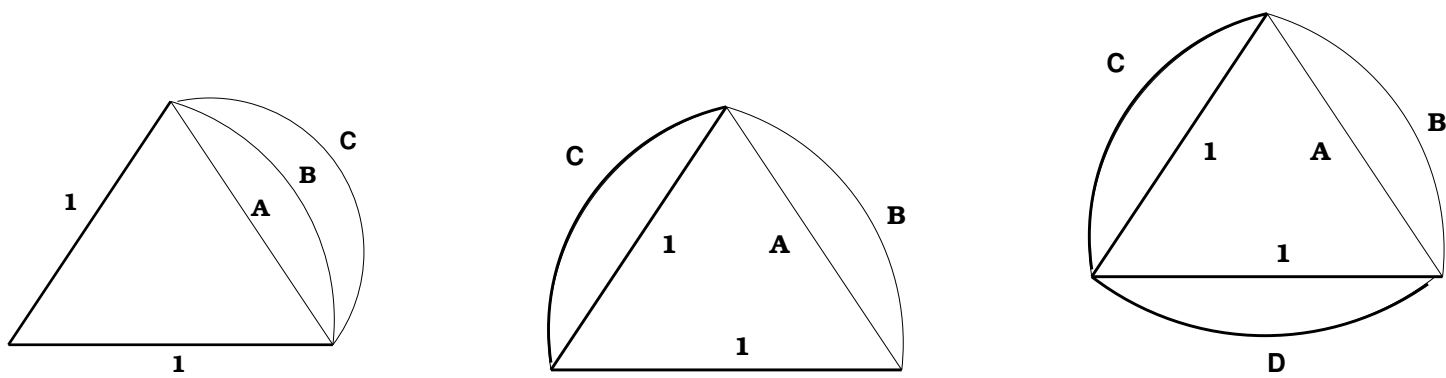

FiguRE 6. Triangle graphs with possibly multiple edges
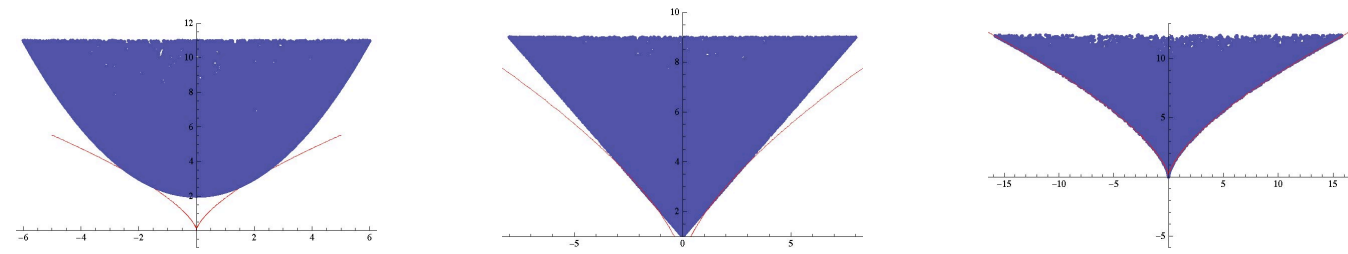

FiguRE 7. Spanning tree and characteristic region for the triangle graphs

As Morse singularities are of type $A_{1}$ it is a necessary condition from the above is that there is an $A_{1}$ singularity in the fiber, e.g. via the methods given above. For a Dirac point, one in addition needs to check the signature.

3.4. Forced degeneracies by Symmetries. One reason that singular points have to be present is given by symmetries. If the momentum space geometry stems from a graph, such symmetries can be induced by symmetries of the underlying graph. The procedure for this is not straightforward though and proceeds via re-gauging groupoid and a "lift" of its action to the momentum space [24]. The result of this rather elaborate process is the existence of projective representations of subgroups of the symmetry group of the graph that appear as stabilizers in the geometric action on the momentum space. We describe this construction below.

In the geometric examples of wire networks, we showed in [24] that all the singularities of the Eigenvalue cover are forced by these enhanced re-gauging symmetries.

3.4.1. General setup. Going back to the embedding of $\mathscr{B}_{\Theta}$ into $M_{k}\left(\mathbb{T}_{\Theta}^{n}\right)$ the relevant matrix representation depended on the choice of a rooted spanning tree $\left(\tau, v_{0}\right)$ and an order $<$ on the vertices. We will now fix that the first element in that order is given by the root. In [24] we showed that the re-gauging from $(\tau,<)$ to $\left(\tau^{\prime},<^{\prime}\right)$ is given by conjugation by a unitary matrix $U_{\tau,<}^{\tau^{\prime},<^{\prime}}$. These matrices are more complicated than just the permutation group and incorporate local gaugings. These are given by diagonal matrices with invertible elements in $\mathbb{T}_{\Theta}$ indexed by the vertices of the graph.

Moreover in this way, the automorphism group of $\Gamma$ acts by re-gaugings. Namely, if $\phi \in \operatorname{Aut}(\Gamma)$ then given $(\tau,<)$, the image of $\tau, \phi(\tau)$, and the push forward of the order, $\phi_{*}(<)$, give rise to a re-gauging by $U_{\tau,<}^{\phi(\tau), \phi_{*}(<)}$. Usually this action on a given Hamiltonian is not trivial, due to the fact that $\rho$ need not be trivial.

All these observations directly generalize to the more general case of a groupoid representation $(\bar{\Gamma}, \rho)$. In this case $\mathbb{T}_{\Theta}$ is replaced by $\mathscr{T}$. The arguments of $[24]$ are not sensitive to the particular 
structure of $\mathbb{T}_{\Theta}$ and hence carry over to the more general situation. We summarize the logical steps here.

3.4.2. Re-gauging groupoid. The re-gaugings form a secondary groupoid, the re-gauging groupoid. Its objects are given by tuples $(\tau,<)$ and between any two objects there is a unique morphism $\left((\tau,<),\left(\tau^{\prime},<^{\prime}\right)\right)$. There is a morphism $\lambda$ to matrices with coefficients in $\mathscr{T}$ by sending $\left((\tau,<),\left(\tau^{\prime},<^{\prime}\right)\right)$ to $U_{\tau,<^{\tau^{\prime}}<<^{\prime}}$. This morphism need not be a representation, however, since we are only guaranteed that $\lambda\left(g_{1}\right) \lambda\left(g_{2}\right) \lambda\left(g_{1} g_{2}\right)^{-1}$ is a non-commutative 2-cocycle with values in $U(\mathscr{T})$, the unitary elements of $\mathscr{T}$. The reason for this is that under the identification given in $\S 2.2 .5$ the re-gauging basically corresponds to an isomorphism of $\pi_{1}\left(\bar{\Gamma}, v_{0}\right)$ with $\pi_{1}\left(\bar{\Gamma}, v_{0}^{\prime}\right)$ along a path, $v_{0}$ and $v_{0}^{\prime}$ being the roots of $\tau$ and $\tau^{\prime}$ respectively. Concatenating the isomorphisms along these paths as above, we end up with an isomorphism under a loop; but this is precisely conjugation with an element of $\pi_{1}\left(\bar{\Gamma}, v_{0}\right)$. In the representation, this element becomes an element in $U(\mathscr{T})$.

3.4.3. Projective Groupoid Representations. In the commutative case the cocycle above gives rise to a central extension by $U(\mathscr{T})$ and the matrices $U_{\tau,<}^{\tau^{\prime},<^{\prime}}$ give a representation in $M_{k}(\mathscr{T})$ of the central extension.

Evaluating with a character $\hat{\chi}$, the extension becomes an extension by $U(1)$ and the matrices $\hat{\chi}\left(U_{\tau, \dot{\tau^{\prime}},<}\right)$ form a projective representation of the groupoid in $M_{k}(\mathbb{C})$.

3.4.4. Stabilizer Groups, Lifts, Projective Actions and Group Extensions. If we have a fixed point, that is a Hamiltonian that is invariant under the action of non-trivial groupoid elements, then these elements form a group of re-gaugings. Technically the representation of stabilizer subgroupoid factors through the group given by identification of all objects in that groupoid to one point.

In order to find such a stabilizer group, we look for an automorphism of $T$ which compensates the re-gauging by automorphisms of $\bar{\Gamma}$. That is, given an automorphism $\phi$ of $\bar{\Gamma}$, let $\Phi_{\tau^{\prime},<^{\prime}}^{\tau,<}$ be the associated re-gauging. We then look for an automorphism $\Psi_{\tau^{\prime},<^{\prime}}^{\tau,<}$ of $T$ such that

$$
\hat{\chi}_{t}\left(\Phi_{\tau^{\prime},<^{\prime}}^{\tau,<}\left(H_{\tau,<}\right)\right)=\hat{\chi}_{\Psi_{\tau^{\prime},<^{\prime}}^{\tau,<}(t)}\left(H_{\tau,<}\right)
$$

This is done for one orbit of $(\tau,<)$ under $\operatorname{Aut}(\bar{\Gamma})$. This tool is most effective if the graphs are completely symmetric, like the cases we considered.

If we find such a lift of the automorphism group $\operatorname{Aut}(\bar{\Gamma}) \rightarrow \operatorname{Aut}(T)$, then we can look for points of enhanced symmetry. If $t \in T$ has a non-trivial stabilizer group under this action of $\operatorname{Aut}(\bar{\Gamma})$ then the matrix $\hat{\chi}_{t}\left(H_{\tau,<}\right)$ has a non-trivial re-gauging fixed group. This action by conjugation yields a projective representation of the stabilizer group.

Given such a projective representation, we know that it is a representation of a central $U(1)$ extension of the stabilizer group. If the stabilizer group is finite, we would furthermore like to find a smaller if possible finite group which already carries the representation. That is an extension of the stabilizer group by a finite group. For this one uses the theory of Schur multipliers.

The upshot is that the isotypical decomposition of the representation has to be commensurate with the Eigenspace decomposition of the Hamiltonian - for that particular value $t \in T$. Practically this means that on the one hand if in the given representation there are irreps of dimension bigger than one, one can infer that there are degeneracies in the spectrum of at least these dimensions. On the other hand, the one dimensional isotypical components fix Eigenvectors and hence make it easy to find the Eigenvalues. In general of course one only has to diagonalize the Hamiltonian inside the isotypical summands. 
3.5. Invariants of the momentum space geometry. In the commutative case, a last way to characterize the singularities in the momentum space geometry is to use topological invariants. These come from the fact that although the cover $X \rightarrow T$ is unramified and trivial outside of $T_{d e g}$ the line bundles defined by each non-degenerate Eigenvalue carry non-trivial topology.

3.5.1. Basic bundles, and their K-theoretic and Cohomology Valued Charges. More precisely, let $X_{\mathrm{deg}}=\pi^{-1}\left(T_{\mathrm{deg}}\right)$ be the closed singular locus of $X$. Then the restriction

$$
\pi: X_{0}:=X \backslash X_{\mathrm{deg}} \rightarrow T_{0}:=T \backslash T_{\mathrm{deg}}
$$

is the trivial $k$-fold cover, since the Eigenvalues are real. A trivialization is given by choosing the order in each fiber according to the order in $\mathbb{R}$ of the Eigenvalues $\lambda_{1}<\cdots<\lambda_{k}$.

On this restriction the $C^{*}$-algebra $C_{0}\left(X \backslash X_{\mathrm{deg}}\right)$ contains pairwise orthogonal projections $P_{i}$ such that $H=\sum_{i} \lambda_{i} P_{i}$. Each of these $P_{i}$ defines a rank 1 sub-bundle $L_{i}$ of the trivial bundle $X_{0} \times \mathbb{C}$ which is the Eigenbundle corresponding to the Eigenvalue $\lambda_{i}$. The projector or equivalently the bundle $L_{i}$ defines an element in $K$-theory $\left[L_{i}\right] \in K\left(T_{0}\right)$. We will continue with the geometric interpretation of line bundles and $\mathrm{K}$-theory here, although in a forthcoming analysis we will concentrate on the $C^{*}$ version of $K$-theory in oder to move to a non-commutative setup.

We call the classes $\left[L_{i}\right]$ the $\mathrm{K}$-theoretic charges and the associated Chern classes

$$
\beta_{i}:=c_{1}\left(L_{i}\right) \in H^{2}\left(T_{0}\right)
$$

the cohomological charges. We also let $C=\bigoplus_{i} L_{i}$, and $[C] \in K\left(T_{0}\right)$ be its class in $K$-theory. Finally we define the polynomial invariant $Q_{c}\left(t_{i}\right)=\prod_{i}\left(1+t_{i} \beta_{i}\right) \in H^{\mathrm{ev}}\left(T_{0}\right)\left[t_{i}\right]$. This class contains all the cohomological information of the $L_{i}$ and $C$.

Remark 3.11. One can generalize most of the arguments to non-Hermitian variations: $H: T \rightarrow G L(k, \mathbb{C})$, but then one should impose that $T_{0}$ is simply connected and $\pi_{1}\left(T_{0}\right)=1$ in order for the characteristic polynomial to be irreducible over $C_{0}\left(T_{0}\right)$ which is necessary to define the $P_{i}$, see e.g. [17].

Remark 3.12. We assumed that the Hamiltonians are generically non-degenerate. It is sufficient to assume that the ranks of the Eigenbundles are generically constant. In this case, we have vector bundles $V_{i}$ and total Chern classes $c\left(V_{i}\right)$.

3.5.2. Numerical Invariants/Charges. One can try to get numerical information about $Q_{c}$ and the $\beta_{i}$ by pairing them with appropriate homology classes. For this it is easier to assume that we are dealing with oriented manifolds. If we furthermore have a differentiable structure, we know that we can evaluate Chern classes by using Chern-Weil theory.

The paring then corresponds to the integral of the curvature form for any connection over a submanifold of the correct even degree. The set of all such numbers on a set of generators of homology of $T_{0}$ then determines the cohomological charges as functions on homology. By the classification theorem for line bundles, see e.g. [20] the first Chern class fixes the isomorphism class of the line bundle. Furthermore, if we use at least $\mathbb{Q}$ coefficients, usually in physics we take $\mathbb{R}$ of $\mathbb{C}$, cohomology and K-theory are isomorphic via the Chern character and we can represent homology by using submanifolds [20,35]. Notice that by the results of Thom [35] all second homology classes are of this type even over $\mathbb{Z}$.

It then follows that the charges are trivial if $T_{0}$ has vanishing second cohomology, which is where the first Chern classes live, (e.g. if $T_{0}$ is 2 -connected). In that case the Chern classes $\beta_{i}$ vanish and the line bundles $\left[L_{i}\right]$ are trivializable. This is the case in some examples, notably the honeycomb. The effect of the line bundles being trivializable is that the associated points of degeneracy are not topologically stable, see $§ 3.6$. 
The two-torus or the two-sphere do however have non-vanishing $H^{2}$ and thus are prime candidates to detect first Chern classes if we embed them into the momentum space.

In this particular case, we can evaluate the first Chern class of a line bundle with a connection on a 2-dimensional submanifold by pulling back, i.e. restricting, the line bundle to the surface and integrating the curvature form of the connection. Here if $A$ is a connection form for the line bundle, and the first Chern-class is represented by the curvature form $\Omega=d A+\frac{1}{2} A \wedge A$.

Explicitly, if $\Sigma$ is an oriented compact surface and $i: \Sigma \rightarrow T$ is an embedding, then

$$
Q_{\Sigma, i}:=\int_{\Sigma} i^{*} c_{1}\left(L_{i}\right)=\left\langle c_{1}\left(L_{i}\right), i_{*}([\Sigma])\right\rangle
$$

where $\langle$,$\rangle is the standard pairing between cohomology and homology.$

3.5.3. Berry connection. It was Berry's [7] great insight in this context, that adiabatic transport provides such a connection and that this connection is indeed not always trivial and produces the so-called Berry phase as a possible monodromy.

Simon [34] realized that Berry's formula is just the calculation of the first Chern class of $\left[L_{i}\right]$ using a connection and Chern-Weil theory. Thus one can use any other connection, for instance the so-called canonical connection used by Simon. By general theory the first Chern class is the only obstruction for the line bundle, and hence the monodromy, to be trivial.

3.5.4. Standard Setup. Let us first fix the concrete setup which is usually present. Assume T is compact orientable potentially with boundary and that $T_{\operatorname{deg}}$ is in codimension at least 1 ; i.e. $T$ is generically non-degenerate. We furthermore assume that $T_{\operatorname{deg}} \cap \partial T=\emptyset$. Then $T_{0}$ is an orientable manifold with boundary. Let $N$ be a tubular neighborhood of $T_{\operatorname{deg}}$ in $T$ and $\bar{N}$ its closure. $^{3}$ Then $B=T \backslash N$ is a compact sub-manifold with boundary $\partial B=\partial T \amalg \partial \bar{N}$ where $\partial \bar{N}=\bar{N} \backslash N$.

E.g. If we assume that $T_{\text {deg }}$ is a manifold with singularities and the smooth part of $T_{\text {deg }}$ is of codimension $r$ then $\partial \bar{N}$ is an $S^{r-1}$ bundle over the smooth part of $T_{\mathrm{deg}}$. In particular, if $T_{\mathrm{deg}}$ is a discrete set of points $p_{i}$ we can take $N$ to be the union of small open balls centered at each point and $\bar{N}$ will be the union of the closed balls, while $\partial N$ will be the union of the corresponding spheres.

3.5.5. Even dimensional $B$. If $B$ is even dimensional, we can pair with $B$ and consider $\int_{B} Q_{c}\left(t_{i}\right)$.

If in particular $T_{0}=T$ and $T$ is two-dimensional then $B=T$ and we obtain all the individual charges by using $B=\Sigma$ as $Q_{i}:=\int_{B} \beta_{i}$.

Following Simon [34] this if for instance the case for the quantum Hall effect. Here $T=T^{2}$ has no degenerate locus and we have that $B=T$ can carry non-trivial line bundles. Indeed the arguments of TKNN [37] establish the non-triviality of the corresponding line bundle.

3.5.6. Odd dimensional $T$ with boundary. If $T$ is odd dimensional, we can restrict the $L_{i}$ to the boundary $\partial T$, since we assumed that $\partial T \cap T_{\text {deg }}=\emptyset$. Then the boundary charge is defined to be $\left.\int_{\partial T} Q_{c}(t)\right|_{\partial T_{0}}$.

In the differentiable case, we represent $Q_{c}$ by a closed form $\omega=d \phi$ of even degree; strictly speaking this is a polynomial form. Also, since $B$ is odd dimensional, we have by Stokes' Theorem

\footnotetext{
${ }^{3}$ I.e. $N$ is a smooth submanifold of the same dimension as $T$, which can be deformation retracted onto $T_{\text {deg. }}$. If $T_{\mathrm{deg}}$ is smooth $N$ can be chosen to be a standard small tubular neighborhood. Otherwise we assume that $T_{d e g}$ is sufficiently nice that we have a stratification that allows for Thom-Mather theory [36, 31].
} 
that $0=\int_{B} \omega=\int_{\partial B} \phi=\int_{\partial T} \phi+\int_{-\partial \bar{N}} \phi$.

$$
\int_{\partial T} \phi=-\int_{-\partial \bar{N}} \phi=\int_{\partial \bar{N}} \phi
$$

where $\partial \bar{N}$ has the outward orientation viewed from $\bar{N}$. Else we just use the usual pairing between the corresponding homologies and cohomologies.

If the boundary is empty, then we have that $\int_{\partial \bar{N}} \phi=0$.

3.5.7. Local charges and codimension 3. For each component $\bar{N}_{k}$ of $\bar{N}$, we can consider the restriction $i_{\partial \bar{N}_{i}}^{*}\left(Q_{c}\right)$ of $Q_{c}$ to $\partial N_{k}$, where $k$ indexes the components, and define the local charge of that component to be $\int_{\partial \bar{N}_{k}} i_{\partial \bar{N}_{i}}^{*}\left(Q_{c}\right)$. This is of course only useful if $T$ is odd-dimensional, so that $\partial \bar{N}$ is even-dimensional.

A special situation arises, if the smooth part $T_{\mathrm{deg}}^{\mathrm{sm}}$ of $T_{\mathrm{deg}}$ is of codimension 3 . Recall that $\partial \bar{N}$ is an $S^{r-1}$ bundle over $T_{\mathrm{deg}}^{\mathrm{sm}}$, where $r=\operatorname{codim}\left(T_{\mathrm{deg}}^{\mathrm{sm}}\right)$ which in this case is 3 and hence $\partial \bar{N}$ is a 2 -sphere bundle. Thus in this case, we can restrict the $L_{i}$ to the fiber $S^{2}=S^{2}(p)$ over any point $p$ of $T_{\mathrm{deg}}^{\mathrm{sm}}$. We call $\left.\int_{S^{2}(p)} \beta_{i}\right|_{S^{2}}(p)$ the $i$-th local charge at $p$ and $\left.\int_{S^{2}(p)} Q_{c}\right|_{S^{2}}(p)$ the local charge.

3.5.8. Isolated critical points in dimension 3 . For isolated critical points of $T_{\mathrm{deg}}$ the local charges are just given by integrating over small spheres around these points, which is what $\partial \bar{N}$ is. If $T_{\text {deg }}$ consists only of isolated critical points, then formula (9) states that the boundary charge is the sum over the local charges. If moreover the boundary is empty, this means that the sum of all the $\mathrm{i}$-th local charges is 0 . This is the case for the gyroid.

3.5.9. Slicing. A slicing for $T$ is a smooth codimension 1 foliation by compact oriented manifolds of $T$ which has a global transverse section $S$ and the leaves of the foliation generically do not intersect $T_{\text {deg. }}$. For this we need the Euler characteristic to be 0, which is in particular the case for all odd dimensional compact manifolds.

For $s \in S$ let $F_{s}$ be the leaf of $s$ and $i_{s}$ be the inclusion, if $F_{s} \cap T_{d e g}=\emptyset$, we can consider the pullback of $C$ and consider

$$
Q_{s}:=\int_{F_{s}} i^{*} Q_{c}
$$

which is the total Chern class of the slice. An interesting commonly encountered situation arises if

(1) $F_{s}$ generically does not intersect $T_{\text {deg }}$

(2) Each component of $\bar{N}$ contains only one component for $T_{\mathrm{deg}}$.

(3) Any component of $\bar{N}$ is contained between some pair of slices. That is for a component $T_{\operatorname{deg}}^{\prime} \subset T_{\operatorname{deg}}$ there are $s_{1}, s_{2}$ and an n-dimensional closed submanifold $M$ of the $\mathrm{n}-$ dimensional manifold $T$, such that $M \cap T_{\mathrm{deg}}=T_{\mathrm{deg}}^{\prime}$, and $\partial M \cap T_{\mathrm{deg}}=\emptyset, \partial M=F_{s_{1}}-F_{s_{2}}$ and the component of $\bar{N}$ corresponding to $T_{\mathrm{deg}}^{\prime}$ is entirely contained in $M$.

In this case, by using Stokes' Theorem we get that the total contribution of $T^{\prime}$

$$
\left.\int_{\partial \bar{N} \cap M} Q_{s}\right|_{\partial \bar{N} \cap M}=Q_{s_{1}}-Q_{s_{2}}
$$

Now (12) is a great tool to numerically find $T_{\mathrm{deg}}$. For this one just runs through the $s \in S$ and looks for jumps in $Q_{s}$. 
3.5.10. $T_{\text {deg }}$ of codimension 3 . If $T^{\prime}$ is smooth then the total charge is

$$
\int_{T_{\text {deg }}}\left(\left.\int_{S^{2}(p)} Q_{c}\right|_{S^{2}}(p)\right) d p=Q_{s_{1}}-Q_{s_{2}}
$$

If we are in dimension 3 then codimension 3 means that the degenerate locus consists of only isolated critical points. Here the equation (12) simplifies to just a finite sum over the critical points.

If furthermore the critical points are $A_{1}$ singularities, see $\S 3.3 .1$, then the jumps in the charge are from \pm 1 to $\mp 1$, as calculated in $[34,17]$ depending on if one calculates for the upper or lower band and the chosen orientation/parameterization.

3.5.11. 3-dimensional torus models. If we have that $T=T^{3}$ the situation is especially nice. It is fibered by $T^{2}$ s via any of the three projections $\pi_{j}: S^{1} \times S^{1} \times S^{1} \rightarrow S^{1}, j=1,2,3$. The inclusion of fibers of the three coordinate projections actually generates the whole cohomology of $T^{3}$. It has non-vanishing 2 nd cohomology $H^{2}\left(T^{3}\right) \simeq \mathbb{Z}^{3}$. In contrast to the two-torus where puncturing kills the 2nd cohomology a punctured three torus actually still has second cohomology. It is given explicitly in the proof of the theorem below. This is a main difference between graphene and the gyroid, see below. One has to be sure however, that the condition of generically not intersecting the degenerate locus is not violated. A counterexample is the case for the $D$-surface, see below. Given finitely many points $p_{i} \in T^{3}$, we say that they are in generic position with respect to an identification $T^{3} \simeq S^{1} \times S^{1} \times S^{1}$, if all their coordinates (viz. projections) are pairwise distinct. By changing the identification with automorphisms of $T^{3}$, we can always obtain this situation.

Notice that the slicing along any of the three co-ordinate foliations given by the projections $\pi_{j}, j=1,2,3$ only gives a finite set of numbers $Q_{s, i}$ for each Eigenbundle $L_{i}$, since the integral over the Chern-class is constant as $s$ varies in a component of $S^{1} \backslash\left\{\pi_{j}\left(p_{i}\right)\right\}$.

Theorem 3.13. For a smooth variation with base $T^{3}$ and only finitely many degenerate points, which we may assume to be in generic position, the slicing method applied to all three coordinate projections completely determines the $K$-theoretic charges and hence the line bundles $L_{i}$ up to isomorphism.

Proof. For no degenerate points this is clear as $H^{2}\left(T^{3}, \mathbb{Z}\right) \simeq \mathbb{Z}^{3}$ and generators are given by the three coordinate embeddings of $T^{2}$.

If there are $m \geq 1$ degenerate points $p_{i}$ and pick a coordinate projection $\pi_{j}$ and let

$$
z_{1}, \ldots, z_{m} \in S^{1}
$$

be the images of the $p_{i}$. Let $t_{1}, \ldots, t_{m}$ be points in between the $z_{i}$, that is one point per component of $S^{1} \backslash\left\{z_{i}\right\}$. Consider the CW model of the torus, which has one 2-cell at height $t_{i}$ and 3-cells in between and 0 and 1 cells accordingly. Then $T_{0}=T \backslash\left\{p_{i}\right\}$ deformation retracts onto the 2-skeleton of this complex. And the homology of $T_{0}$ can be calculated either (a) via the standard Meyer-Vietoris sequence for $T$ covered by $N$ and a slightly enlarged $B$, or (b) using cellular chains for the above CW complex. Using the former, we see that there are $m-1+3$ classes in $H^{2}\left(T_{0}, \mathbb{Z}\right) \simeq \mathbb{Z}^{m+2}$. Namely the three original classes, plus the classes of the $m$ little spheres minus the diagonal class of all the spheres. In the CW basis of (b) this is given by a set of $m$ horizontal slices separating the $m$ points and the images of the two other coordinate embeddings of $T^{2}$.

Now the slicing method will give the paring with these two cells and as the Poincaré paring is non-degenerate, the cohomology class of $c_{1}\left(L_{i}\right)$ is determined by these numbers and hence the line bundle up to isomorphism. 

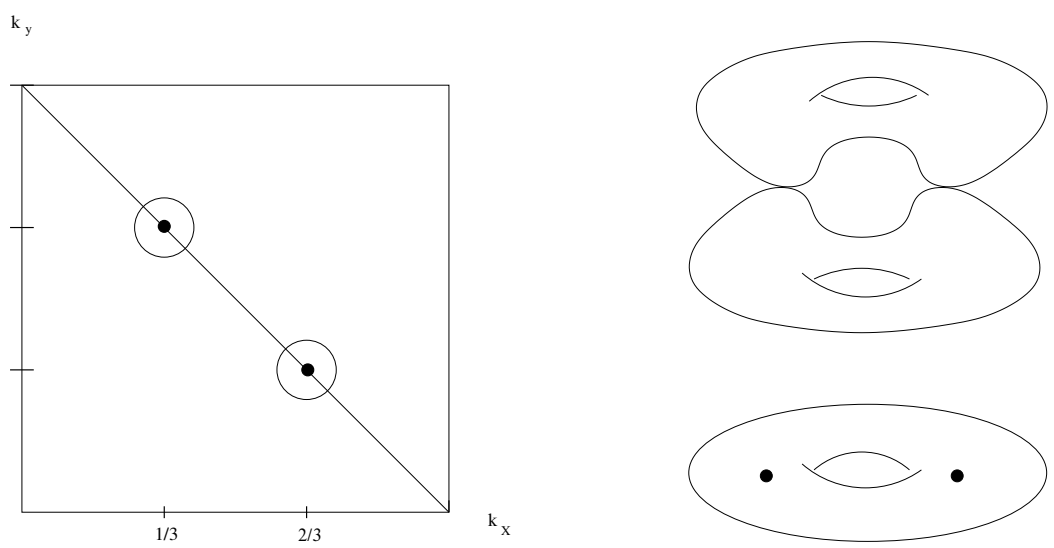

Figure 8. I. The base two torus with the singular points (as a square with opposite sides identified), the anti-diagonal $(\phi, \bar{\phi})$, the 2 points of $T_{d e g}$ and the discs making up $N$. II. A picture of the ramified double cover $X$.

Remark 3.14. In fact, one only needs one complete slicing and then one slice each in the other coordinate directions as determining data.

3.6. Topological Stability. Having non-vanishing topological charges produces topological stability. If we perturb the Hamiltonian slightly by adding a small perturbation term $\lambda H_{1}$ and continuously vary $\lambda$ starting at 0 , then $T_{0}$ does not move much - for instance as a submanifold of $T \times R$, see §3.3.1. In particular, there will be no new singular points in $T_{0}$ for small perturbation. The Eigenbundles over $T_{0}$ also vary continuously and hence so do their Chern classes. Since these are defined over $\mathbb{Z}$ they are actually locally constant, so that all the non-vanishing charges, scalar, K-theoretic or cohomological, must be preserved.

\section{Results for the COMmutative AND NON-COMmutative $C^{*}$-GeOMetries of WiRE NETWORKS}

In this section, we summarize our results for the different quantum wire networks, honeycomb, $\mathrm{P}$ (or more generally any Bravais lattice), D and G. The basis are the results from [21, 22, 23, 24] and a new analysis for the topological charges using slicing.

The first set of results are on the singular geometry of the momentum space in the commutative situation. These include all the three aspects developed above, the branched cover and its singularities, the symmetries and the topological invariants.

The second set of results are on the classification of the $C^{*}$-algebras that appear when one allows a constant background magnetic field.

\subsection{Singular geometry of the momentum space for periodic wire networks.}

4.1.1. The Honeycomb Lattice. In this case the space $X$ is a double cover of the torus $T^{2}$ ramified at two points $\left(e^{2 \pi i \frac{1}{3}}, e^{-2 \pi i \frac{1}{3}}\right)$ and $\left(e^{-2 \pi i \frac{1}{3}}, e^{2 \pi i \frac{1}{3}}\right)$. These two points are $A_{1}$ singularities and Dirac points. This is depicted in Figure 8. $T_{0}$ is $T^{2}$ with two points removed, so $H^{2}\left(T_{0}\right)=0$ and so all the charges vanish and all bundles are trivial, thus the two Dirac points are in general not topologically stable. Along the anti-diagonal $(\phi, \bar{\phi})$ we have the equation for the two sheets of the cover $E(\phi)= \pm(1+2 \cos (\phi))$. This is depicted in Figure 9 . 


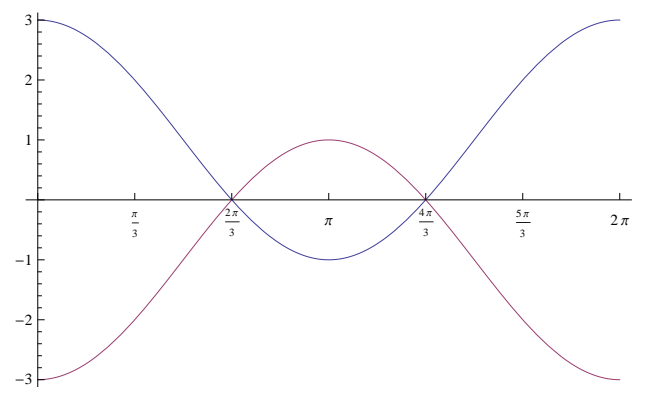

Figure 9. The cover along the anti-diagonal $(\phi, \bar{\phi})$

Remark 4.1. There has been an investigation of deformation directions which do not destroy these points [12]. In our setup this means the following: the characteristic map has its image in $[-9,0]$ where the swallowtail for $A_{1}$ is the point 0 . One only considers deformations which still have 0 in the image of the characteristic map.

At the Dirac points there is an enhanced symmetry which is Abelian, so it does not have any higher dimensional irreps, but the isotypical decomposition is fully decomposed and forces the double degeneracy at the Dirac points due to the form of the Hamiltonian.

4.1.2. The primitive cubic (P) case, and other Bravais cases. The cover $X \rightarrow T^{k}$ is trivial and so is the line bundle of Eigenvectors.

Remark 4.2. The analysis of [5] of the quantum Hall effect, however, suggests that there is a non-trivial noncommutative line bundle in the case of $k=2$ for non-zero B-field. Furthermore, in this case there is a non-trivial bundle, not using the noncommutative geometry, but rather the Eigenfunctions constructed in [37] for the full Hilbert space $\mathscr{H}$. This is what is also considered in [34]. We will study this phenomenon in the gyroid and the other cases in the future.

4.1.3. The Diamond $(D)$ case. In this case, we see that that $1-\chi(\Gamma)=3$ and $T$ is the $3-$ Torus $T^{3}$. The space $X$ defined by $\mathscr{B}$ in the commutative situation is a generically 2 -fold cover of $T^{3}$ where the ramification locus $T_{\mathrm{deg}}$ is along three circles on $T^{3}$ given by the equations $\phi_{i}=\pi, \phi_{j} \equiv \phi_{k}+\pi \bmod 2 \pi$ with $\{i, j, k\}=\{1,2,3\} . T_{\text {deg }}=\Xi^{-1}(0)$ is the inverse image -of the characteristic map - of the only singular point (the origin) of the miniversal unfolding of $A_{1}$. The characteristic region is the interval $[-16,0]$.

Thus the singularities are of type $A_{1}$ but they are not discrete, but rather pulled back to the entire $T_{\mathrm{deg}}$, hence there are also no Dirac points. Figure 10 depicts the base 3 -torus with the singular locus, which is of codimension 2.

The space $T_{0}=T^{3} \backslash T_{\mathrm{deg}}$ contracts onto a 1-dimensional CW-complex and hence has $H^{2}\left(T_{0}\right)=0$. Thus there are no non-vanishing topological charges associated to this geometry and no stability.

Analogous to the honeycomb case there are Abelian enhanced symmetries with 1-dimensional isotypical components, which force the double degeneracy in view of the structure of the Hamiltonian.

4.1.4. The Gyroid $(G)$ case. For the gyroid, the commutative geometry is given by a generically unramified 4 -fold cover of the three torus, see [21]. There are only 4 ramification points. This means that the locus is of real codimension 3 contrary to the $\mathrm{D}$ case where it was of codimension 2. Furthermore the degenerations are 3 branches coming together at 2 points $-(0,0,0)$ and 


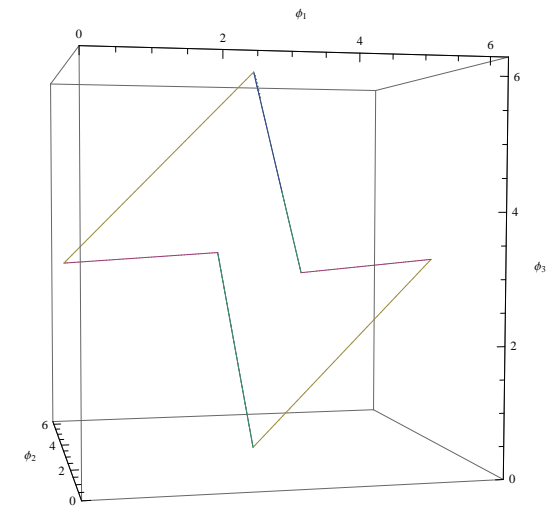

Figure 10. The base 3 -torus as a cube with opposite sides identified and the singular locus consisting of three $S^{1}$ s mutually intersecting in two points

$(\pi, \pi, \pi)$ - and 2 pairs of branches coming together at the other two points $-\left(\frac{\pi}{2}, \frac{\pi}{2}, \frac{\pi}{2}\right)$ and $\left(\frac{3 \pi}{2}, \frac{3 \pi}{2}, \frac{3 \pi}{2}\right)$. The latter furnish double Dirac points.

Using the characteristic map the first type of singular point corresponds to an $A_{2}$ singularity and the second type corresponds to the type $\left(A_{1}, A_{1}\right)$ stratum of the swallowtail. All the inverse images have discrete fibers. There are two image points on the $A_{2}$ stratum each with one inverse image under $\Xi$ and there is one point on the $\left(A_{1}, A_{1}\right)$ stratum, with two inverse images.
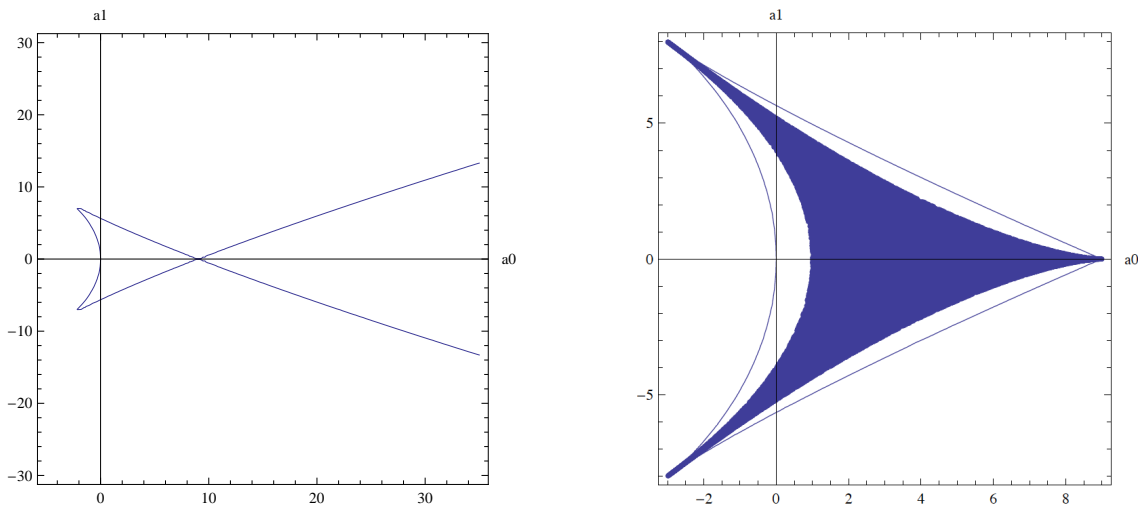

Figure 11 . The -6 slice of the swallowtail of $A_{3}$ and the region occupied by the gyroid

All the $A_{1}$ singularities in the fibers are Dirac points. That is there are four of these points. Furthermore at all points there are enhanced symmetries by non-Abelian groups.

At $(0,0,0)$ the enhanced symmetry group is the symmetric group $\mathbb{S}_{4}$ - the full symmetry group of $\bar{\Gamma}$ which entirely lifts to $A u t\left(T^{3}\right)$ - yielding one 1-dim irrep and one 3-dim irrep which forces the triple degeneracy. At $(\pi, \pi, \pi)$ we have an a priori projective representation of $\mathbb{S}_{4}$, which we showed however to be equivalent to the standard representation of $\mathbb{S}_{4}$ and hence we again get one 1-dim irrep and one 3-dim irrep which forces the triple degeneracy. At the other two points things are really interesting. The stabilizer symmetry group is $A_{4}$ and it yields a projective representation which is carried by the double cover of $A_{4}$ aka. $2 A_{4}, 2 \mathrm{~T}$, the binary 

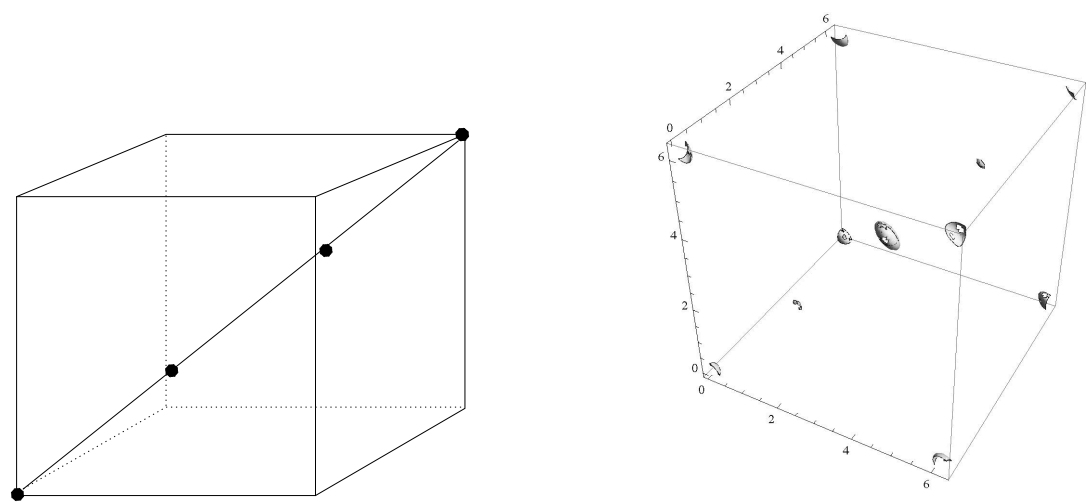

Figure 12. The 4 points of $T_{\text {deg }}$ along the diagonal (again $T^{3}$ is depicted as a cube with opposite sides identified) and the 4 spheres making up $\partial \bar{N}$

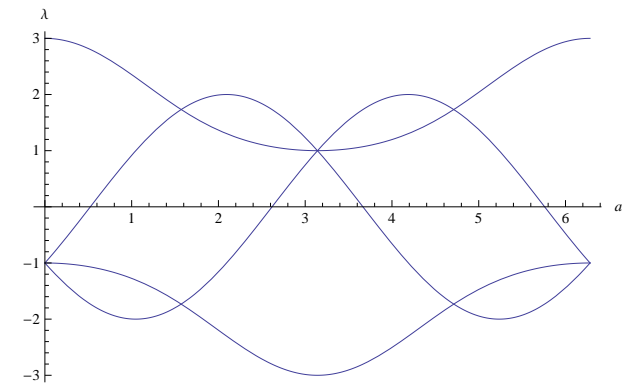

FIGURE 13. The spectrum/cover along the diagonal

tetrahedral group or $S L(2,3)$. The representation decomposes into two 2-dim irreps forcing the two double degeneracies.

Notice that we essentially need a projective representation, since $A_{4}$ itself has no 2-dim irreps.

Now $T_{\text {deg }}$ is the set of the four points above and $T_{0}=T^{3} \backslash T_{\operatorname{deg}}$ contracts onto a $2-\operatorname{dim}$ CW complex with non-trivial second homology.

Thus there are $K$-theoretic and cohomological charges. This is the special case of dimension 3 with codimension 3 degenerate points and moreover we have a slicing of $T^{3}$ by the fiber bundle $T^{3} \rightarrow S^{1}$ by any of the tree coordinate projections. In fact the homology is generated by any four slices which sit in between the 4 slices that contain the degenerate points. Pairing with these surfaces completely determines the Chern class of the line bundles and hence the line bundles up to isomorphism.

Figure 12 depicts $T^{3}$ with the 4 singular point as well as the 4 spheres making up $\partial \bar{N}$. Figure 13 shows the cover along the diagonal of $T^{3}$ which contains $T_{\text {deg }}$.

Figure 14 shows the different slices which on the one hand make up the CW complex and on the other give the submanifolds the curvature is integrated over to yield the $Q_{s, i}$. The relevant numerics were carried out in [25].

The result of the numerical slicing is contained in Figure 15 as well as the analytic values. 


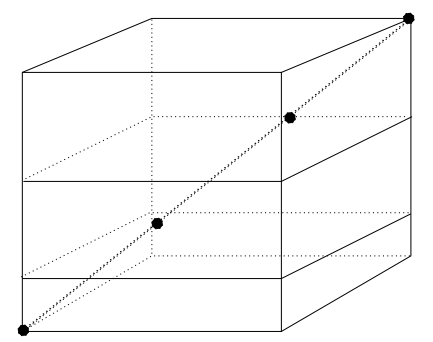

FIGURE 14. Slices corresponding to the first coordinate projection
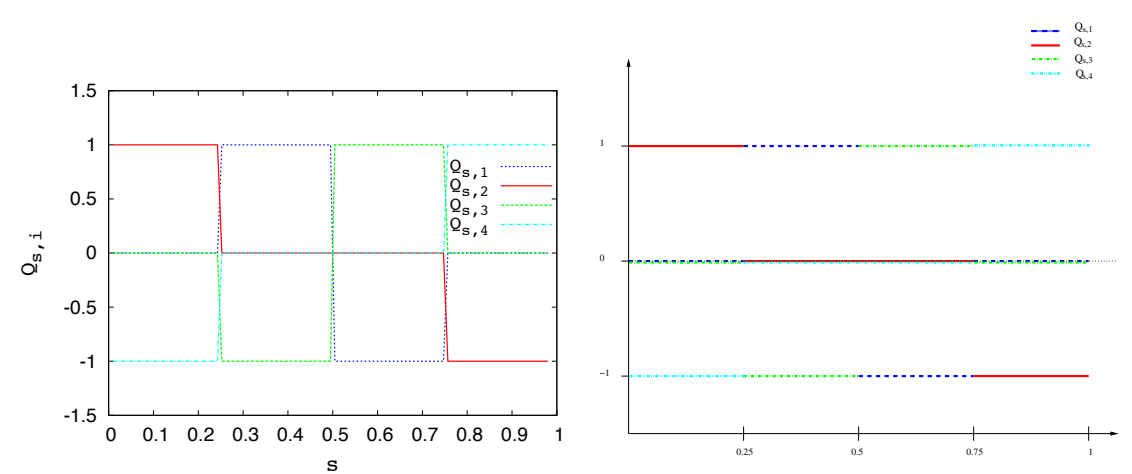

Figure 15. The charges $Q_{s, i}, i=1, \ldots 4$ as functions of $s$, the position of the slice (compare to Fig. 14). Left: the result of a numerical computation. Right: the analytical values

In accordance with the analytic calculations of $[34,17]$ the Dirac points yield jumps in the charge by \pm 1 for the two bands that cross. A new result is that the $A_{2}$ points yield jumps by $-2,0,2$ for the three bands that cross. This behavior is typical of a standard example of a 3-dimensional degenerating family of Hamiltonians with a single triple crossing considered in $[7,34]$ and we conjecture that indeed locally the family of the Gyroid is diffeomorphic to this family.

All these charges are topologically stable. In preliminary numerical simulations introducing symmetry breaking deformations we found that the $A_{2}$ points each split into four $A_{1}$ points in compliance with the jumps given above. We expect to explain this behavior using time reversal symmetry.

4.2. Results on the non-commutative geometry. In this section we summarise our results for the non-commutative geometry of the PDG, Bravais and Honeycomb wire networks, resulting form a constant magnetic field $B$ (see 2.1.2 and [21, 22]).

4.2.1. Honeycomb. Generically $\mathscr{B}_{\Theta}=\mathbb{T}_{\Theta}^{2}$. In order to give the degenerate points, let

$$
-e_{1}:=(1,0), e_{2}=\frac{1}{2}(1, \sqrt{3}), e_{3}:=\frac{1}{2}(1,-\sqrt{3})
$$

be the lattice vectors and $f_{2}:=e_{2}-e_{1}=\frac{1}{2}(-3, \sqrt{3}), f_{3}:=e_{3}-e_{1}=\frac{1}{2}(3, \sqrt{3})$ the period vectors of the honeycomb. The parameters we need are

$$
\theta:=\hat{\Theta}\left(f_{2}, f_{3}\right), \quad q:=e^{2 \pi i \theta} \quad \text { and } \quad \phi=\hat{\Theta}\left(-e_{1}, e_{2}\right), \quad \chi:=e^{i \pi \phi}, \text { thus } \quad q=\bar{\chi}^{6}
$$


where $\hat{\Theta}$ is the quadratic from corresponding to the $B$-field $B=2 \pi \hat{\Theta}$.

Theorem 4.3. [21] The algebra $\mathscr{B}_{\Theta}$ is the full matrix algebra of $M_{2}\left(\mathbb{T}_{\theta}^{2}\right)$ except in the following finite list of cases

(1) $q=1$.

(2) $q=-1$ and $\chi^{4}=1$.

The precise algebras are given in [21]. We wish to point out that $q=\chi=1$ is the commutative case and $q=-\chi=1$ is isomorphic to the commutative case, while the other cases give noncommutative proper subalgebras of $M_{2}\left(\mathbb{T}_{\theta}^{2}\right)$.

4.2.2. $P$ and Bravais cases. For the simple cubic lattice and any other Bravais lattice of rank $k$ ( $\mathrm{P}$ is the rank 3 case): if $\Theta \neq 0$ then $\mathscr{B}_{\Theta}$ is simply the noncommutative torus $\mathbb{T}_{\Theta}^{k}$ and if $\Theta=0$ then this $\mathscr{B}_{0}$ is the $C^{*}$ algebra of $T^{k}$. There are no degenerate points.

4.2.3. Diamond. In the non-commutative case, we express our results in terms of parameters $q_{i}$ and $\xi_{i}$ defined as follows: Set $e_{1}=\frac{1}{4}(1,1,1), e_{2}=\frac{1}{4}(-1,-1,1), e_{3}=\frac{1}{4}(-1,1,-1)$ for $B=2 \pi \Theta$ let

$$
\Theta\left(-e_{1}, e_{2}\right)=\varphi_{1} \quad \Theta\left(-e_{1}, e_{3}\right)=\varphi_{2} \quad \Theta\left(e_{2}, e_{3}\right)=\varphi_{3} \text { and } \chi_{i}=e^{i \varphi_{i}} \text { for } i=1,2,3
$$

There are three operators $U, V, W$, given explicitly in [22], which span $\mathbb{T}_{\Theta}^{3}$ and have commutation relations

$$
U V=q_{1} V U \quad U W=q_{2} W U \quad V W=q_{3} W V
$$

where the $q_{i}$ expressed in terms of the $\chi_{i}$ are:

$$
q_{1}={\overline{\chi_{1}}}^{2} \chi_{2}^{2} \chi_{3}^{2} \quad q_{2}={\overline{\chi_{1}}}^{6}{\overline{\chi_{2}}}^{2}{\overline{\chi_{3}}}^{2} \quad q_{3}={\overline{\chi_{1}}}^{2}{\overline{\chi_{2}}}^{6} \chi_{3}^{2}
$$

Vice versa, fixing the values of the $q_{i}$ fixes the $\chi_{i}$ up to eighth roots of unity:

$$
\chi_{1}^{8}=\bar{q}_{1} \bar{q}_{2} \quad \chi_{2}^{8}=q_{1} \bar{q}_{3} \quad \chi_{3}^{8}=q_{1}^{2} \bar{q}_{2} q_{3}
$$

Other useful relations are $q_{2} \bar{q}_{3}=\bar{\chi}_{1}^{4} \chi_{2}^{4} \bar{\chi}_{3}^{4}$ and $q_{2} q_{3}=\bar{\chi}_{1}^{8} \bar{\chi}_{2}^{8}$. the algebra $\mathscr{B}_{\Theta}$ is the full matrix algebra except in the following cases in which it is a proper subalgebra.

(1) $q_{1}=q_{2}=q_{3}=1$ (the special bosonic cases) and one of the following is true:

(a) All $\chi_{i}^{2}=1$ then $\mathscr{B}_{\Theta}$ is isomorphic to the commutative algebra in the case of no magnetic field above.

(b) Two of the $\chi_{i}^{4}=-1$, the third one necessarily being equal to 1 .

(2) If $q_{i}=-1$ (special fermionic cases) and $\chi_{i}^{4}=1$. This means that either

(a) all $\chi_{i}^{2}=-1$ or

(b) only one of the $\chi_{i}^{2}=-1$ the other two being 1 .

(3) $\bar{q}_{1}=q_{2}=q_{3}=\bar{\chi}_{2}^{4}$ and $\chi_{1}^{2}=1$ it follows that $\chi_{2}^{4}=\chi_{3}^{4}$. This is a one-parameter family.

(4) $q_{1}=q_{2}=q_{3}=\bar{\chi}_{1}^{4}$ and $\chi_{2}^{2}=1$ it follows that $\chi_{1}^{4}=\bar{\chi}_{3}^{4}$. This is a one-parameter family.

(5) $q_{1}=q_{2}=\bar{q}_{3}=\bar{\chi}_{1}^{4}$ and $\chi_{1}^{2}=\bar{\chi}_{2}^{2}$. It follows that $\chi_{3}^{4}=1$. This is a one-parameter family. 
4.2.4. Gyroid. To state the results of [21] we use the bcc lattice vectors

$$
\begin{gathered}
g_{1}=\frac{1}{2}(1,-1,1), \quad g_{2}=\frac{1}{2}(-1,1,1), \quad g_{3}=\frac{1}{2}(1,1,-1) \\
\theta_{12}=\frac{1}{2 \pi} B \cdot\left(g_{1} \times g_{2}\right), \quad \theta_{13}=\frac{1}{2 \pi} B \cdot\left(g_{1} \times g_{3}\right), \quad \theta_{23}=\frac{1}{2 \pi} B \cdot\left(g_{2} \times g_{3}\right) \\
\alpha_{1}:=e^{2 \pi i \theta_{12}} \bar{\alpha}_{2}:=e^{2 \pi i \theta_{13}} \alpha_{3}:=e^{2 \pi i \theta_{23}} \\
\phi_{1}=e^{\frac{\pi}{2} i \theta_{12}}, \quad \phi_{2}=e^{\frac{\pi}{2} i \theta_{31}}, \quad \phi_{3}=e^{\frac{\pi}{2} i \theta_{23}}, \quad \Phi=\phi_{1} \phi_{2} \phi_{3}
\end{gathered}
$$

\section{Classification Theorem.}

(1) If $\Phi \neq 1$ or not all $\alpha_{i}$ are real then $\mathscr{B}_{\Theta}=M_{4}\left(\mathbb{T}_{\Theta}^{3}\right)$.

(2) If $\Phi=1$, all $\alpha_{i}= \pm 1$, at least one $\alpha_{i} \neq 1$ and all $\phi_{i}$ are different then $\mathscr{B}_{\Theta}=M_{4}\left(\mathbb{T}_{\Theta}^{3}\right)$.

(3) If $\phi_{i}=1$ for all $i$ then the algebra is the same as in the commutative case.

(4) In all other cases (this is a finite list) $\mathscr{B}$ is non-commutative and $\mathscr{B}_{\Theta} \subsetneq M_{4}\left(\mathbb{T}_{\Theta}^{3}\right)$.

\section{OutLOOK}

5.1. Observation and conjecture. Looking at the cases above, we observe several regularities. First and foremost, there is agreement on the dimension of the degenerate locus in $T^{k}$ between the commutative and the non-commutative case. In the commutative case, this locus is $T_{\operatorname{deg}} \subset T^{k}$; in the non-commutative case, it is the locus $T_{\mathrm{deg}}^{n c} \subset T^{k}$ of values of the $B$-field, where the matrix algebra is not the full matrix algebra. Here $T^{k}$ parameterizes the entries of $\Theta \bmod \mathbb{Z}$, which parameterize the non-commutative tori.

We conjecture that this is always the case.

There are several possible points of attack here. The first is through the symmetries: as we have seen, the re-gauging groupoid exists already in the non-commutative case. Another is to consider how, in the presence of a conserved topological charge, larger representations, such as $A_{2}$ in the gyroid case, break into smaller pieces. Using the slicing method described above, one can readily see how that happens under a deformation of the Hamiltonian in the commutative case. The question is whether the effect of non-commutativity is something similar.

5.2. Stability, local structure and perturbations. We furthermore plan to analyze the topological invariants further by studying local models for the crossings. In three dimensions, a double crossing has a unique local model up to orientation as already remarked in $[7,34]$. In loc. cit. there are also examples of three-dimensional families with a unique singular point that corresponds to an $n$-fold crossing. Given the jumps in Chern classes we conjecture that for the Gyroid near the triple crossing the family of Hamiltonians restricted to the three bands that are involved is indeed diffeomorphic to that standard family. If this is established, we can show using an additional symmetry argument that the Chern class functions $Q_{s}$ for the slicing and hence the whole geometry of line bundles is entirely determined by the singularities. A further study will then be how higher (more than double) topologically protected crossings dissolve under perturbations.

\section{ACKNOWLEDGMENTS}

RK thankfully acknowledges support from NSF DMS-0805881 and DMS-1007846. BK thankfully acknowledges support from the NSF under the grants PHY-0969689 and PHY-1255409. Any opinions, findings and conclusions or recommendations expressed in this material are those of the authors and do not necessarily reflect the views of the National Science Foundation. This 
work was partially supported by grants from the Simons Foundation (\#267481 to Erika Birgit Kaufmann and \#267555 and collaboration grant \#317149 to Ralph Kaufmann). Both RK and BK thank the Simons Foundation for this support. They also thank M. Marcolli for insightful discussions.

Parts of this work were completed when RK and BK were visiting the IAS in Princeton and the Max-Planck-Institute in Bonn, and RK was visiting the IHES in Bures-sur-Yvette and the University of Hamburg with a Humboldt fellowship. We gratefully acknowledge all this support.

\section{REFERENCES}

[1] A. A. Agrachev, On the Space of Symmetric Operators with Multiple Ground States, Funct. Anal. and its App. 45, 241-251 (2011)

[2] V. I. Arnold, V.V. Goryunov, O.V. Lyashko and V.A. Vasil'ev, Singularity Theory I, Springer, Berlin Heidelberg, 1998

[3] V. I. Arnol'd, Remarks on eigenvalues and eigenvectors of Hermitian matrices, Berry phase, adiabatic connections and quantum Hall effect. (English summary), Selecta Math. (N.S.) 1 (1995) 119

[4] J. Bellissard, Gap labelling theorems for Schrödinger operators, in: From number theory to physics (1992) 538-630. DOI: 10.1007/978-3-662-02838-4_12

[5] J. Bellissard, A. van Elst and H. Schulz-Baldes, The noncommutative geometry of the quantum Hall effect. Topology and physics, J. Math. Phys. 35 (1994) 5373-5451. DOI: 10.1063/1.530758

[6] B. A. Bernevig, T. L. Hughes, S. Raghu and D. P. Arovas, Theory of the Three-Dimensional Quantum Hall Effect in Graphite, Phys. Rev. Lett. 99 (2007) 146804. DOI: 10.1103/PhysRevLett.99.146804

[7] M. V. Berry, Quantum phase factors accompanying adiabatic changes, Proc. R. Soc. Lond. A 392 (1984) 45-57. DOI: 10.1098/rspa.1984.0023

[8] A. H. Castro Neto, F. Guinea, N. M. R. Peres, K. S. Novoselov, and A. K. Geim, The electronic properties of graphene, Rev. Mod. Phys. 81 (2009) 109. DOI: 10.1103/RevModPhys.81.109

[9] A. Connes, Noncommutative Geometry. Academic Press, Inc., San Diego, CA, 1994.

[10] M. Demazure, Classification des germs à point critique isolé et à nombres de modules 0 ou 1 (d'après Arnol'd). Séminaire Bourbaki, 26e année, Vol. 1973/74 Exp. No 443, pp. 124-142, Lect. Notes Math. 431. Springer, Berlin 1975

[11] V. Ya. Demikhovskii and D. V. Khomitskiy, Quantum Hall effect in p-type heterojunction with a lateral surface superlattice, Phys. Rev. B 68 (2003) 165301. DOI: 10.1103/PhysRevB.68.165301

[12] C.F. Fefferman, M.I. Weinstein, Honeycomb Lattice Potentials and Dirac Points, preprint. arXiv:1202.3839

[13] D.Gieseker, H.Knoerrer, E.Trubowitz, An overview of the geometry of algebraic Fermi curves. in "Algebraic geometry: Sundance 1988", 19-46, Contemp. Math., 116, Amer. Math. Soc., Providence, RI, 1991.

[14] N. Goldman and P. Gaspard, Quantum graphs and the integer quantum Hall effect, Phys. Rev B 77 (2008) 024302. DOI: 10.1103/PhysRevB.77.024302

[15] J. Goryo and M. Kohmoto, Berry Phase and Quantized Hall Effect in Three-Dimension, J. Soc. Jpn. 71 (2002) 1403. DOI: 10.1143/JPSJ.71.1403

[16] K. Grosse-Brauckmann and M. Wohlgemuth, The gyroid is embedded and has constant mean curvature companions, Calc. Var. Partial Differential Equations 4 (1996) 499. DOI: 10.1007/BF01261761

[17] K. Grove and G. K. Pedersen, Diagonalizing Matrices over C(X), J. Funct. Anal. 59 (1984) 65-89. DOI: $10.1016 / 0022-1236(84) 90053-3$

[18] D. A. Hajduk et. al., The gyroid - a new equilibrium morphology in weakly segregated diblock copolymers Macromolecules 27 (1994) 4063-4075. DOI: 10.1021/ma00093a006

[19] P.G. Harper, Single band motion of conduction electrons in a uniform magnetic field, Proc. Phys. Soc. London A68 (1955) 874-878. DOI: 10.1088/0370-1298/68/10/304

[20] D. Husemöller, Fibre Bundles, Graduate Texts in Mathematics (Book 20), Springer, 1993

[21] R.M. Kaufmann, S. Khlebnikov, and B. Wehefritz-Kaufmann, The geometry of the double gyroid wire network: quantum and classical, Journal of Noncommutative Geometry 6 (2012) 623-664. DOI: $10.4171 / \mathrm{JNCG} / 101$

[22] R.M. Kaufmann, S. Khlebnikov, and B. Wehefritz-Kaufmann, The noncommutative geometry of wire networks from triply periodic surfaces, Journal of Physics: Conf. Ser. 343 (2012) 012054. DOI: 10.1088/1742-6596/343/1/012054

[23] R.M. Kaufmann, S. Khlebnikov, and B. Wehefritz-Kaufmann, Singularities, swallowtails and Dirac points. An analysis for families of Hamiltonians and applications to wire networks, especially the Gyroid. Annals of Physics 327 (2012) 2865. DOI: 10.1016/j.aop.2012.08.001 
[24] R.M. Kaufmann, S. Khlebnikov, and B. Wehefritz-Kaufmann, Re-gauging groupoid, symmetries and degeneracies for Graph Hamiltonians and applications to the Gyroid wire network, accepted for publication in AHP (2015). arXiv:1208.3266

[25] R.M. Kaufmann, S. Khlebnikov, and B. Wehefritz-Kaufmann, Topologically stable Dirac points in a threedimensional supercrystal. In preparation.

[26] S. Khlebnikov and H. W. Hillhouse, Electronic Structure of Double-Gyroid Nanostructured Semiconductors: Perspectives for carrier multiplication solar cells, Phys. Rev. B 80 (2009) 115316. DOI: 10.1103/PhysRevB.80.115316

[27] M. Koshino, H. Aoki, K. Kuroki, S. Kagoshima and T. Osada, Hofstadter Butterfly and Integer Quantum Hall Effect in Three Dimensions, Phys. Rev. Lett. 86 (2001) 1062. DOI: 10.1103/PhysRevLett.86.1062

[28] C. A. Lambert, L. H. Radzilowski, E. L. Thomas, Triply Periodic Level Surfaces as Models for Cubic Tricontinous Block Copolymer Morphologies, Philos. Transactions: Mathematical, Physical and Engineering Sciences, Vol. 354, No 1715, Curved Surfaces in Chemical Structure (1996) 2009-2023

[29] G. De Nittis and G. Landi, Topological aspects of generalized Harper operators, The Eight International Conference on Progress in Theoretical Physics, Mentouri University, Constantine, Algeria, October 2011; Conference proceedings of the AIP, edited by N. Mebarki and J. Mimouni (2012) 58-65. DOI: 10.1063/1.4715400

[30] M. Marcolli and V. Mathai, Towards the fractional quantum Hall effect: a noncommutative geometry perspective, in: Noncommutative geometry and number theory: where arithmetic meets geometry, Caterina Consani, Matilde Marcolli (Eds.), Vieweg, Wiesbaden (2006) 235-263. DOI: 10.1007/978-3-8348-0352-8_12

[31] J. Mather, Notes on Topological Stability, photocopied notes, Harvard Univ., Cambridge, MA, 1970; Stratifications and mappings, in Dynamical Systems, 195-232, Proc. Sympos., Univ. Bahia (Salvador, Brazil, 1971) (M. M. Peixoto, ed.), Academic Press, New York, 1973.

[32] G. Panati, H. Spohn and S. Teufel, Effective dynamics for Bloch electrons: Peierls substitution and beyond, Comm. Math. Phys. 242 (2003) 547-578. DOI: 10.1007/s00220-003-0950-1

[33] A.H. Schoen, Infinite periodic minimal surfaces without self-intersections, NASA Technical Note No. TN D $-5541(1970)$

[34] B. Simon, Holonomy, The Quantum Adiabatic Theorem, and Berry's Phase, Phys. Rev. Lett. 51, 2167-2170 (1983). DOI: 10.1103/PhysRevLett.51.2167

[35] R. Thom. Sous-variétés et classes d'homologie des variétés différentiables. II. Résultats et applications. C. R. Acad. Sci. Paris 236, (1953). 573-575

[36] R. Thom, Ensembles et morphismes stratifiés, Bull. Amer. Math. Soc. 75 (1969), 240-284 DOI: $10.1090 /$ S0002-9904-1969-12138-5

[37] D.J. Thouless, M. Kohmoto, M.P. Nightingale and M. den Nijs, Quantized Hall Conductance in a TwoDimensional Periodic Potential, Phys. Rev. Lett. 49 (1982) 405-408. DOI: 10.1103/PhysRevLett.49.405

[38] V.N. Urade, T.C. Wei, M.P. Tate and H.W. Hillhouse. Nanofabrication of double-Gyroid thin films. Chem. Mat. 19, 4 (2007) 768-777. DOI: 10.1021/cm062136n

[39] J. von Neumann and E. Wigner, Über das Verhalten von Eigenwerten bei adiabatischen Prozessen, Z. Phys. 30, 467 (1929)

[40] G. Xu, H. Weng, Z. Wang, X. Dai, and Z. Fang, Chern Semimetal and the Quantized Anomalous Hall Effect in $\mathrm{HgCr}_{2} \mathrm{Se}_{4}$, Phys. Rev. Lett. 107 (2011) 186806. DOI: 10.1103/PhysRevLett.107.186806

Ralph M. Kaufmann, Department of Mathematics, Purdue University, West Lafayette, in 47907 E-mail address: rkaufman@math.purdue.edu

Sergei Khlebnikov, Department of Physics and Astronomy, Purdue University, West Lafayette, IN 47907

E-mail address: skhleb@physics.purdue.edu

Birgit Wehefritz-Kaufmann, Department of Mathematics and Department of Physics and Astronomy, Purdue University, West Lafayette, IN 47907

E-mail address: ebkaufma@math.purdue.edu 